\title{
An artificial moth \\ Chemical source localization using a robot based neuronal model of moth optomotor anemotactic search
}

\section{Journal Article}

\section{Author(s):}

Pyk, Pawel; Bermúdez i Badia, Sergi; Bernardet, Ulysses; Knüsel, Philipp; Carlsson, Mikael; Gu, Jing; Chanie, Eric; Hansson, Bill S.; Pearce, Tim C.; Verschure, Paul F.M.J.

\section{Publication date:}

2006-06

\section{Permanent link:}

https://doi.org/10.3929/ethz-b-000036696

\section{Rights / license:}

In Copyright - Non-Commercial Use Permitted

\section{Originally published in:}

Autonomous Robots 20(3), https://doi.org/10.1007/s10514-006-7101-4 


\title{
An artificial moth: Chemical source localization using a robot based neuronal model of moth optomotor anemotactic search
}

\author{
Pawel Pyk • Sergi Bermúdez i Badia • \\ Ulysses Bernardet • Philipp Knüsel • Mikael Carlsson • \\ Jing Gu • Eric Chanie • Bill S. Hansson • \\ Tim C. Pearce · Paul F. M. J. Verschure
}

Published online: 8 June 2006

(C) Springer Science + Business Media, LLC 2006

\begin{abstract}
Robots have been used to model nature, while nature in turn can contribute to the real-world artifacts we construct. One particular domain of interest is chemical search where a number of efforts are underway to construct mobile chemical search and localization systems. We report on a project that aims at constructing such a system based on our understanding of the pheromone communication system of the moth. Based on an overview of the peripheral processing of chemical cues by the moth and its role in the organization of behavior we emphasize the multimodal aspects of chemical search, i.e. optomotor anemotactic chemical search. We present a model of this behavior that we test in combination with a novel thin metal oxide sensor and custom build mobile robots. We show that the sensor is able to detect the odor cue, ethanol, under varying flow conditions. Subsequently we
\end{abstract}

P. Pyk $(\varangle) \cdot$ S. Bermúdez i Badia $\cdot$ U. Bernardet $\cdot$ P. Knüsel ·

P. F. M. J. Verschure

Institute of Neuroinformatics, University and ETH Zurich,

Winterthurerstrasse 190, Zurich, CH-8057, Switzerland

e-mail: ppyk@ini.phys.ethz.ch

P. F. M. J. Verschure

ICREA and Department of Technology,

University Pompeu Fabra, Barcelona, Spain

M. Carlsson · B. S. Hansson

Department of Crop Science, Swedish University of Agricultural

Sciences, PO Box 44, Sundsvagen 14, SE-230 53 Alnarp, Sweden

J. Gu · T. C. Pearce

NeuroLab, Centre for Bioengineering, Department of

Engineering, University of Leicester University Road,

Leicester LE1 7RH, UK

E. Chanie

Alpha MOS,

10 Av Didier Daurat, Z1 Montaudran,

31400 Toulouse, France show that the standard model of insect chemical search, consisting of a surge and cast phases, provides for robust search and localization performance. The same holds when it is augmented with an optomotor collision avoidance model based on the Lobula Giant Movement Detector (LGMD) neuron of the locust. We compare our results to others who have used the moth as inspiration for the construction of odor robots.

Keywords Electronic noses - Odor discrimination ·

Mobile olfaction · Gas distribution mapping · Gas source localization

\section{Introduction}

Chemical search has been implemented on robots both to study how biological systems accomplish this task and to develop new real-world applications (Russell, 1999; Ishida et al., 2001). Robotic applications may make significant contributions to environmental monitoring, exploration and chemical source localization. So far, however, the performance of robot based chemical search systems is inferior to their biological counterparts. This is due to both technical and conceptual problems. For instance, the sensitivity observed in nature to chemical compounds is not matched by current chemosensing microtechnology. In addition, odors comprise of complex mixtures, which change dynamically in time, requiring further neuronal processing of the chemical signals detected at the periphery. Moreover, the biomechanical systems embodied in insects by far outstrip the capabilities of modern mobile and flying devices.

One of the best studied biological chemical search systems is pheromone communication in the moth (Kennedy and Marsh, 1974). Male moths are capable to detect and localize female moths over up to several hundred meters while 
the latter secrete only minute quantities of attractants (on the order of $n \mathrm{~g} \mathrm{~h}^{-1}$ ), i.e. pheromones, that are mixed in a complex chemical background signals and dispersed in turbulent plumes. Moths solve this task by combining highly specialized olfactory, anemotactic, and visual sensors with specific information processing and behavioral control strategies. We aim at solving the robot chemical search problem by constructing an artifact that is closely based on our understanding of the sensor processing and behavioral control systems of the moth.

\section{Sensor periphery}

The peripheral mechanism of odorant reception involves the binding of an odorant to a receptor site on a sensory neuron (Mombaerts, 2004). Invertebrate antennae and vertebrate noses exhibit superficial morphological differences but the morphology and function of the receptor cells is strikingly similar (Hildebrand and Shepherd, 1997). Olfactory receptor neurons (ORNs) of both vertebrates and invertebrates are bipolar neurons that project directly to the CNS (Firestein, 2001). Dendritic terminals, or cilia, extend into a lipid/aqueous fluid inside sensilla, which acts as the interface between sensory neurons and the environment. Binding of an odor molecule to a receptor site initiates a cascade of events in the dendritic part of the receptor neuron. These second messenger and transductory pathways seem to be similar in phylogenetically diverse species of animals (Ache, 1994; Hildebrand and Shepherd, 1997). Changes in calcium concentrations in the dendrite lead to depolarization of the membrane that eventually evoke action potentials in the soma when the firing threshold of the neuron is exceeded. Action potentials carry information along the axons to the primary olfactory centre of the brain, the antennal lobe in insects or the olfactory bulb in vertebrates. The frequency of the evoked action potentials in a receptor neuron is dependent on the concentration of the stimulus.

Access to the receptors is complicated by the hydrophobic nature of many odorous volatiles. In the case of invertebrates, the transport of a pheromone to the receptor site on the ORNs in a single sensillum is believed to be facilitated by docking to pheromone binding proteins (PBP). These proteins increase the water solubility of the odorants and might be involved in the binding process of odor molecules to receptor sites.

A breakthrough in olfactory research came when a large multigene family coding for odorant receptor proteins in rats was discovered (Buck and Axel, 1991). Since this historical landmark putative odorant receptor proteins have been identified in a number of organisms (Mombaerts, 1999). The sizes of the gene families coding for these receptors are remarkable and the number of different receptors expressed in olfactory tissues can be as large as 1300 in the mouse (Zhang and
Firestein, 2002). Odorant receptors are G-protein coupled 7transmembrane proteins with little homology between phylogenetically divergent groups of organisms (Mombaerts, 1999). Generally, each ORN expresses only a single type of receptor, although this is currently a topic of controversy (Mombaerts, 2004). It is still not known, however, how odor molecules interact with receptor proteins.

Despite the large number of olfactory receptors a combinatorial strategy is used to detect and discriminate the enormous repertoire of potential odor molecules. Most odor molecules are detected by several different receptors and each receptor can recognize different odorants (Malnic et al., 1999). It seems, however, that each receptor binds a specific set of features, or odotopes, of a molecule (Araneda et al., 2000). That is, different odorants sharing the same odotope bind to the same receptor. It appears that the olfactory system achieves a large coding capacity by exploiting hundreds of overlapping and differently tuned receptors. Here we deploy a thin film metal oxide sensor technology that shares this broad tuning with biological receptors (see methods).

\section{Behavior}

At typical flow velocities in natural open environments, chemical plumes, such as those tracked by moths, show complex filamentous structures (Murlis et al., 2000). The animals' flight velocity combined with the short feature length of the plume micro-structural properties mean that timeaveraged statistics relating, for instance to chemical signal mean, variance, or probability density, cannot be reliably computed in the short time span available. The computation of such measures would require integration times far longer than available to the animal, since the rate of convergence of these measures at this time scale has been shown to be low (Qian and Cowen, 2002). Rather, moths are known to code and exploit behaviorally the instantaneous temporal structure of the plume dynamics (Kennedy et al., 1978). Thus, the instantaneous sensory signals received at chemosensory structures of the moth antenna may well hold key information relating to the nature of the source, its direction and distance, as well as other properties (Murlis et al., 2000; Webster and Weissburg, 2001).

The male moth solves the complex chemical search problem by combining active sampling with a specific behavioral search strategy, where crosswind casting is combined with upwind surges to follow the pheromone plume to its source (Kennedy et al., 1978; Cardé and Hagaman, 1979; Baker and Kuenen, 1982; Vickers and Baker, 1994). This behavior is controlled by specialized chemical detection and orientation systems. The orienting system uses visual cues to control overall navigation relative to wind direction and objects. It has been suggested that the moth relies on the longitudinal and transversal movement of its optic flow to assess 
ground speed and wind direction (Ludlow et al., 1982). For this reason this behavior has been characterized as optomotor anemotaxis (Kennedy and Marsh, 1974). The system that controls the turns and their amplitude during the search is called self-steered counterturning (Kuenen and Baker, 1983).

In addition to processing odor cues and localizing their source, male moths also need to adjust their search trajectory to properties of the environment, such as obstacles and potential predators. Similarly, a robot moth will need to combine its search behavior with avoidance maneuvers due to obstacles or predators. Moreover, flying platforms will have to augment this with flight stabilization maneuvers to compensate for drift and turbulence induced perturbations.

The organization of the visual systems of flying insects is rather species invariant. The hierarchical organization of the different layers of this system feed into a number of neurons that are specialized in detecting specific properties of the visual world, such as optic flow and rapidly approaching surfaces (Egelhaaf and Borst, 1993; Hatsopoulos et al., 1995). Here we will focus on obstacle avoidance using a model of the locust Lobullar Giant Movement Detector neuron (Bermúdez i Badia et al., 2005).

How different sensing and motor control systems are integrated in the moth brain is not fully understood. One of the goals of our modeling study is to explore different scenarios on sensor fusion and behavior selection. Here we will consider the simplest scenario where the optomotor and anemotactic systems are considered as two parallel competitive processing streams that are integrated at the level of the motor ganglion. In this integration the optomotor system will override actions triggered by the anemotactic system. This is based on the observation that visual cues are necessary to trigger and maintain anemotactic search (Kennedy and Marsh, 1974; Charlton and Cardé, 1990).

A number of other groups have also reported moth based or inspired robot applications that perform chemical search (e.g., Kuwana et al., 1999; Lilienthal et al., 2003; Rutkowski et al., 2004). Kuwana et al. use the ElectroAntennoGram (EAG) signal of the antennas of the male silkworm while Lilienthal et al. have presented an alternative approach where a large indoor mobile robot equipped with two groups of three tin-oxide chemosensors each is used for chemical search in a standard office environment $15.4 \times 5.1 \mathrm{~m}$. In addition, Rutkowski et al. study 2 and 3D chemical search using a linear Cartesian gantry to move a sensor in 2 dimensions adding a treadmill to add movements in a third dimension. We will present a more detailed comparison with these approaches in the discussion. In our project (www.amoth.org), we try to technically solve the chemical search and localization problem by developing an Unmanned Aerial Vehicle (UAV) that is based upon our understanding of the moth, i.e. an artificial moth or AMOTH. Here we will present the robot infrastructure that we have developed, the neuronal control models that we have defined for optomotor behavior and chemical search, together with an overview of the biology on which they are based. In particular we will focus on the multi-modal aspects of chemical search. We will analyze the performance of the robots in solving 2 dimensional olfactory search problems in a wind tunnel under varying wind and stimulus delivery conditions. In our experiments, we want to understand how the different components of the anemotactic optomotor control system need to interact to provide reliable and robust navigation and accurate chemical source localization. After evaluating the different components of our system, we will assess the ability of a control system that solely relies on the competition between feedforward motor signals, derived from its different sensory processing modules, to control a mobile robot in a 2 dimensional chemical search and obstacle avoidance task.

\section{Methods}

The artificial moth (amoth) described here consists of a chemosensor system, a mobile or flying platform and a simulated neuronal control system.

The chemosensor

The 6 grid array thin film metal oxide chemosensor used (Fig. 1(a) provides a broad spectrum of sensitivity to a wide range of volatile organic compounds (Alpha MOS SA, France). This technology has relatively low power consumption (approx. $270 \mathrm{~mW}$ ) while providing a high degree of miniaturization. Moreover, selectivity of individual sensors to different compounds can be controlled by its geometric organization as well as variations in the semiconductor materials and dopants used. In this study we used a 6 sensor array where sensors $1-5$ were $n$-type metal oxide semiconductor Tin Oxide $\left(\mathrm{SnO}_{2}\right)$, sensors 2 and 4 were doped with catalytic metal additives Palladium $(\mathrm{Pd})$ and Platinum $(\mathrm{Pt})$ respectively, while sensor 6 was a $p$-type metal oxide semiconductor Niobium Oxide $\left(\mathrm{Nb}_{2} \mathrm{O}_{5}\right)$. The bulk resistance of these chemo sensitive resistors depends upon various physical parameters, such as surface interaction between odor molecules and the semiconductor material (Nanto and Stetter, 2003).

The discrete chemosensor array was integrated on a PCB (Fig. 1(b) that controlled its heating to determine the operating temperature, and sampled the sensor resistance. The sensor resistance was converted to a voltage and subsequently digitized. In general we used the fractional change in conductance (FCG) as our measurant that was calculated on a computer according to: 
a

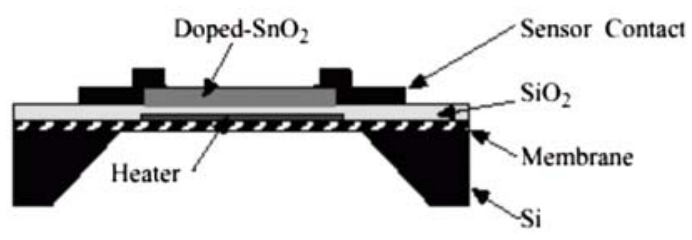

b

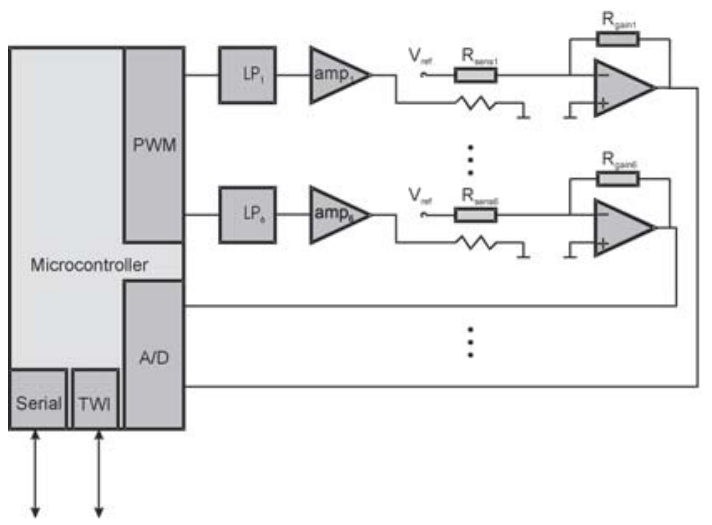

c

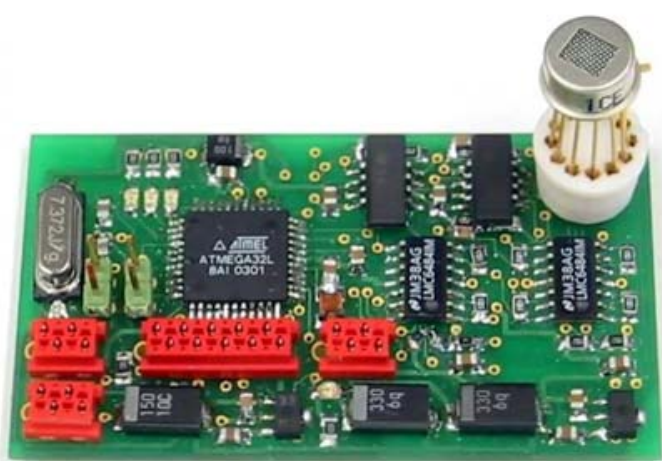

Fig. 1 The AMOTH 6 grid array metal oxide sensor package. (a) Schematic drawing of sensor package indicating its main components. See text for further explanation. Dimensions of single sensor are $0.18 \times 0.2 \mathrm{~mm}$ (with $\times$ length), this creates the active area $0.032 \mathrm{~mm}^{2}$. Thickness of sensitive material is $0.8 \mu \mathrm{m}$ Dimensions of whole 6 grid array sensory chip are $2 \times 3 \times 0.38 \mathrm{~mm}$ (with $\times$ length $\times$ thickness) (b) Application diagram of the chemosensor. PWM signals are generated by the microcontroller are low pass filtered (marked as LP) and amplified (marked as amp), then use for driving the heating elements of the sensor package $\left(R_{\text {heat }}\right)$. The sensor resistance $\left(R_{\text {sens }}\right)$ is converted to a voltage. The board can be interfaced to a host computer via RS232 or TWI bus. (c) Chemosensory board containing a 6 grid array sensor. The sensor sampling rate is around $1045 \mathrm{~Hz}$ that are averaged over 64 samples rendering an effective sampling frequency of $16.3 \mathrm{~Hz}$ for each of the 6 sensory channels. Weight: $13.8 \mathrm{~g}$, dimensions: $34.5 \times 60 \mathrm{~mm}$

$\operatorname{FCG}(t)=\frac{G_{\text {stimulus }}(t)-G_{\text {nostimulus }}}{G_{\text {nostimulus }}}$

where $G_{\text {stimulus }}(t)$ is the conductance $(1 / R)$ during the experiment i.e. in the presence of chemical stimuli and $G_{\text {nostimulus }}$ is the mean conductance obtained under the same conditions as those during experiment but in the absence of any stimuli- this allows us to both calibrate the sensor system to the "real world conditions" as well as to sensor drift.

\section{The AMOTH robots}

For the wind tunnel experiments we have developed a number of custom Plexiglas circular robots with a diameter of $20 \mathrm{~cm}$ (Fig. 2). For locomotion it uses two active wheels (diameter $49 \mathrm{~mm}$ ) placed on the axis of the robot, allowing in

a

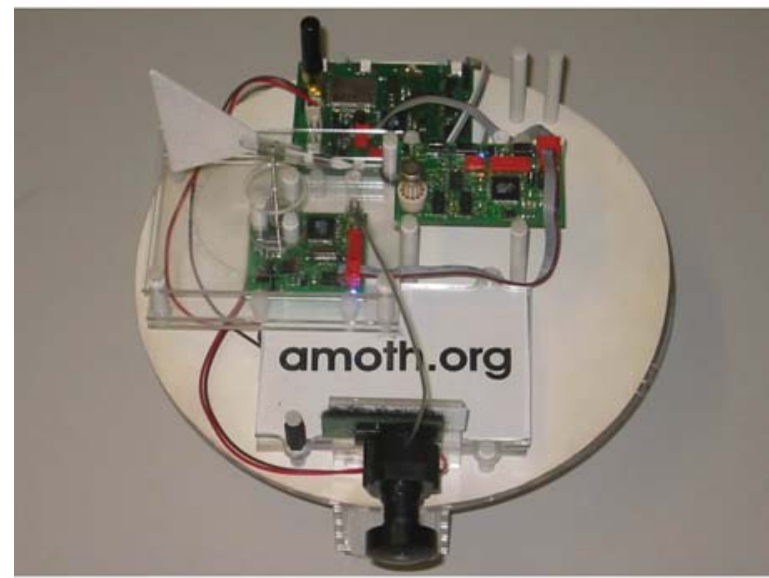

b

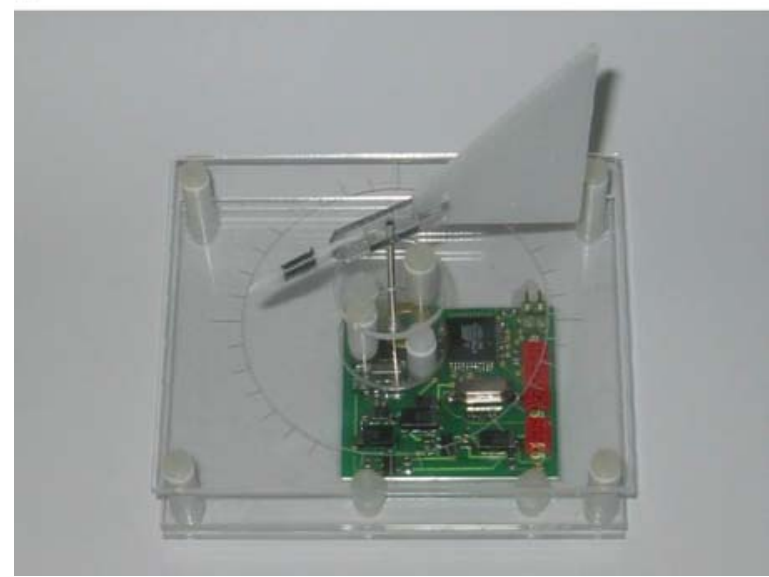

Fig. 2 AMOTH mobile robot platform. (a) AMOTH chemosensory vehicle (diameter $20 \mathrm{~cm}$, height $16 \mathrm{~cm}$ ). Visible are, from top to bottom, control board with Bluetooth communication module, the chemosensor board and the wind direction sensor. On the front of the robot a wireless CMOS color camera is mounted that is fitted with a fisheye lens that provides for a 190 degree field of view. (b) Wind direction sensor, an electronic wind vane sensor consists of a light weight styrophore vane that is attached to a rotating shaft fitted with a small magnet. Dimensions are $3 \mathrm{~mm} \times 3 \mathrm{~mm}$ (diameter $\times$ length). The angular position of the shaft was measured using a 2-axial magnetometer (MicroMag2, PNI Corporation, Santa Rosa, USA, www.pnicorp.com). The shaft and part of the suspension were extracted from a handheld wind speed measuring device (ELV Hand-Windmesser, ELV Elektronik AG, Möhlin, Germany, www.elv.de). The board is fitted with ATmega32L microcontroller that calculates the wind direction and interfaces the magnetometer to the robot infrastructure via the TWI bus 
place rotation, each driven by geared motors with a continuous rotation servo (Parallax-Futaba Continuous Rotation Servo, Parallax, Rocklin, CA, USA, www.parallax.com). The internal motor controllers of the servos were removed and the servos were used as geared motors driven by the external motor controller located on our control board. To provide balance, a free moving metal ball encapsulated in a holder, i.e. caster wheel, was used as the third support point. The chemosensor board was placed in the middle of the platform so that rotations would not affect the position of the chemosensor. The wind direction was measured with a custom build sensor consisting of a wind vane that was fitted to a magnetic encoder (the angular position of the shaft was measured using a 2-axial magnetometer) that was placed near to the chemosensor.

The design of the mobile amoths involved issues of wireless communication, modularity, platform portability, and energy autonomy. Communication is assured by wireless movement control, sensor telemetry and a separate wireless vision system (Fig. 3(a). The movement commands and integrated sensory data are exchanged using a Bluetooth module (Bluetooth $^{\mathrm{TM}}$ RS232 Class I Industrial Adapter, LinTech, Berlin, Germany, www.lintech.de). The robot is equipped with a wireless CMOS color camera (ZT-802A, ZTV Technology Co., Ltd, Shenzhen, China, www.ztv.cc) in the front fitted with a fisheye lens providing for a 190 degree field of view (ORIFL190-3, Omnitech Robotics International LLC, Englewood, USA, www.omnitech.com). The robot is controlled by the neural simulator program IQR (Bernardet et al., 2002) via a wireless link with a ground station. IQR allows us to control the robots with large-scale simulations of insect neuronal systems that are running on a PC or laptop (under the Linux operating system). This solution keeps the onboard computation to a minimum while not compromising the computational requirements of the neuronal systems that we study.

The robot electronics consists of three custom built boards (Fig. 3(b). The olfactory board containing an array of six broadly tuned chemosensors (Fig. 1), and the wind direction sensor (Fig. 2(b) that are each served by their own microcontroller. A third microcontroller is responsible for communication and motor control. All of these microcontrollers (ATmega32L, Atmel, San Jose, CA, USA, www.atmel.com) are connected to a common bus (TWI-Two-Wires Interface) which allows for easy system expansion with any other board, e.g. for our outdoor systems modules additional modules for GPS, 3D compass and altimeters are included. The robot uses Lithium-Polymer rechargeable batteries (KOK 3270, Kokam, Kyunggi-do, Korea, www.kokam.com) that provide up to 5 times higher energy per mass unit than classic Nickel Cadmium rechargeable batteries ( 2 cells with a capacity of $3.3 \mathrm{Ah}$, provide $7.4 \mathrm{~V}$ ). As a result, as the robot an autonomy of up to $8 \mathrm{~h}$ can be achieved. Since the batteries

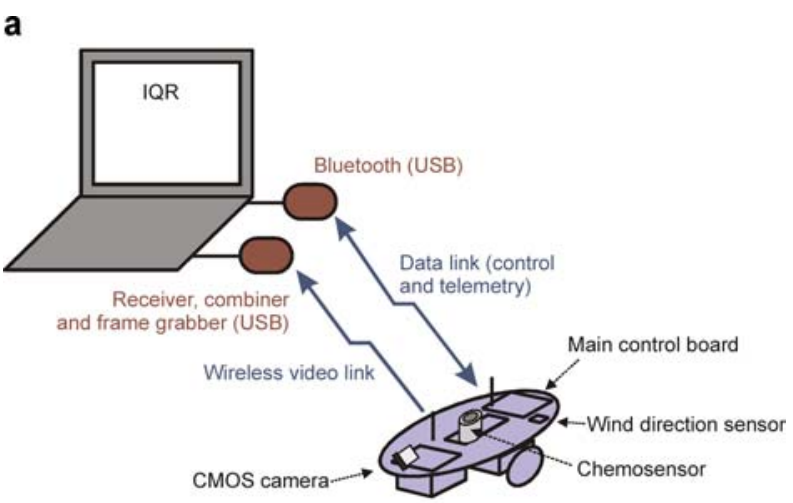

b
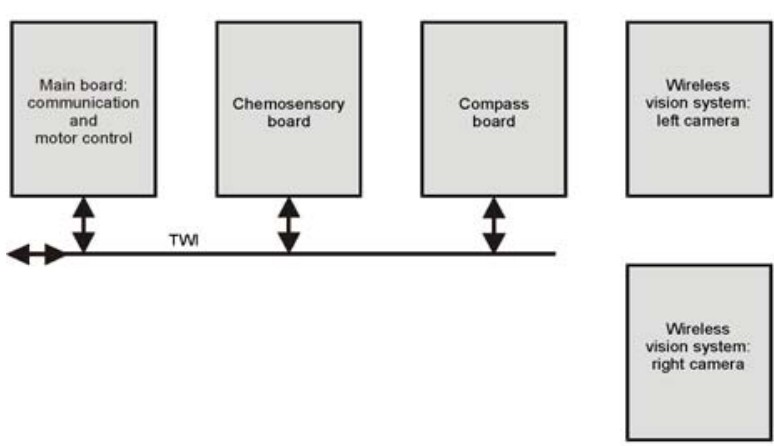

c

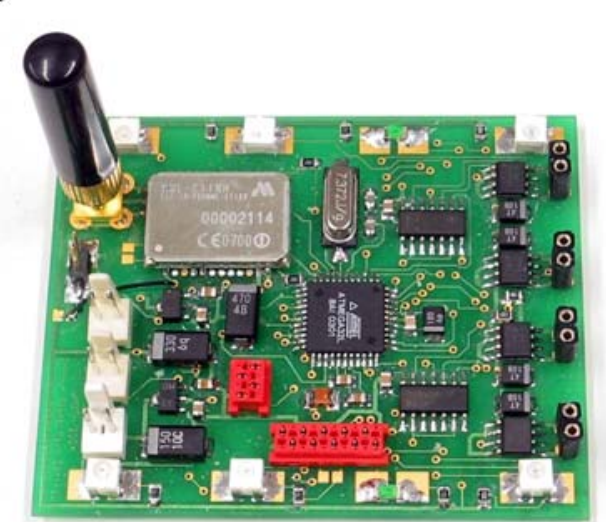

Fig. 3 Experimental setup. (a) The robot setup and its communication with a ground station. See text for further explanation. (b) Diagram of mobile robot control and sensor integration modules. See text for further explanation. (c) Mobile robot control board using a Bluetooth Class I communication module (range up to $100 \mathrm{~m}$ ). Weight: $23.5 \mathrm{~g}$, dimensions: $56 \times 67 \mathrm{~mm}$

are sensitive to discharge and overcharge, the battery status is continuously monitored. The modular architecture of the robot infrastructure was designed to allow easy expansion and application to other vehicles including mobile robots and UAVs.

Wind tunnels

In our experiments we used two wind tunnels. In the first one the responses of the chemosensor array, mounted on 
a wheeled mobile robot platform (Koala, K-team, Lausanne, Switzerland, www.k-team.com), were assessed under varying wind conditions using a wind tunnel (approx. $3 \times 3 \times 0.8 \mathrm{~m}$ (width $\times$ length $\times$ height $)$ ) constructed from transparent polyethylene sheeting. A controllable master $4.4 \mathrm{~kW}$ centrifugal fan generated negative pressure at the outlet of the wind tunnel (of cross-sectional area $3 \times 0.8 \mathrm{~m}$ (length $\times$ height)) to control the flow velocity within the wind tunnel up to $1.0 \mathrm{~m} \mathrm{~s}^{-1}$. Five axial fans were installed at the wind tunnel exit, in front of a manifold feeding the master fan, in order to adjust for a uniform and symmetric velocity profile. Hexcel material of $1 \mathrm{~cm}$ diameter was used at the inlet of the wind tunnel in order to remove large scale eddies from the flow. Ethanol was introduced into the wind tunnel by construction of a platform inside the wind tunnel close to the inlet at the same height as the chemosensor. Absorbent material soaked in chemical stimuli was placed on the platform of $5 \mathrm{~cm}$ in diameter acting as a chemical source. Flow visualization was achieved by filling the wind tunnel with smoke using a smoke generator (Concept Smoke Systems, London, UK) producing approximately $1 \mu \mathrm{m}$ diameter oil/water droplets introduced at the point of the source (nozzle diameter approx. $2.5 \mathrm{~cm}$ ) that were visualized using 4 slide projectors with light slits focused on a single plane positioned at the height of the chemosensor. A camcorder (Panasonic DV 300, Matsushita Electric Industrial, Osaka, Japan) was mounted directly above the wind tunnel for image capture.

All of the mobile robot experiments were performed in a low-cost wind tunnel based on the same principles as the one described above that was constructed from wood and trans-

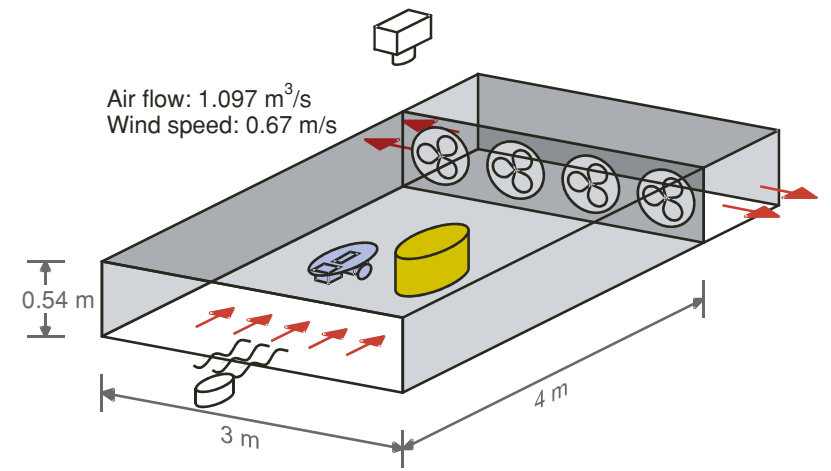

Fig. 4 Structure of the wind tunnel. The wind tunnel is $4 \mathrm{~m}$ long, $3 \mathrm{~m}$ wide and $0.54 \mathrm{~m}$ high. 4 ventilators with independently adjustable speeds at the end of the wind tunnel create negative pressure and suck the air out of the wind tunnel into an exhaust tunnel that is open on both sides. From the exhaust tunnel air is sucked into the exhaust system and removed. The robot (blue) is tracked with a CCD camera mounted above the centre of the wind tunnel. In all experiments, the odor source (white) was placed in the middle at the entrance of the wind tunnel. In some experiments, an obstacle (yellow) was placed inside the wind tunnel. The walls of the wind tunnel were covered with black and white textures to provide visual cues for the optomotor system

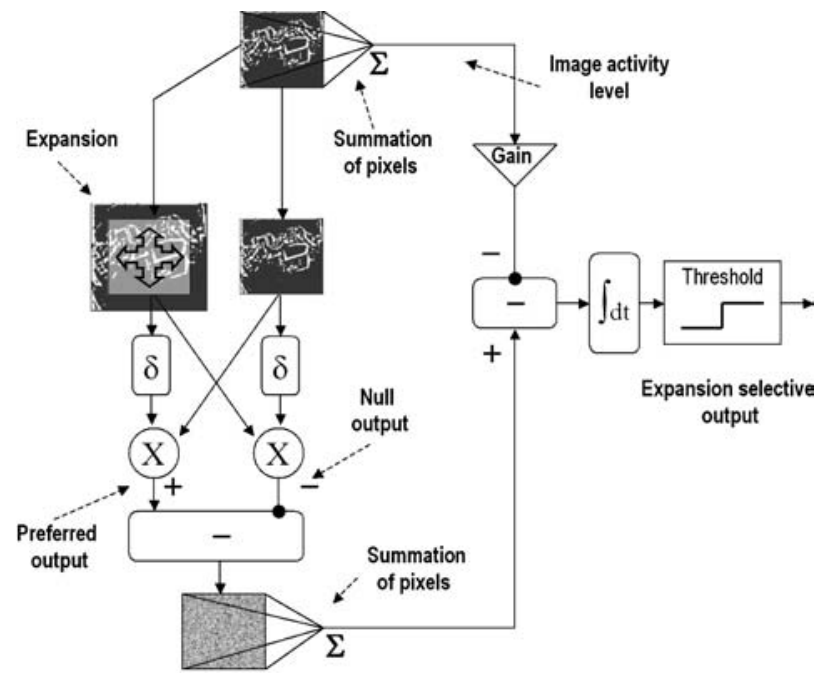

Fig. 5 Implementation of the LGMD model, making use of the Reichardt correlator (Reichardt, 1961), where $\delta$ represents a delay, " $\times$ " the multiplication and " - " the subtraction operation. This model responds to looming stimuli in the visual field normalizing the strength of the response with the global activity level of the image using feed forward inhibition. The specific and non-specific inputs are integrated over time and thresholded (adapted from Bermúdez i Badia and Verschure, 2004)

parent plastic sheets measuring $3 \times 4 \times 0.54 \mathrm{~m}$ (Fig. 4(a)). Solutions of different concentrations of ethanol and distilled water (9.4 and 23.5\% ethanol) were converted using an ultrasonic release system (Mist of Dreams, XrLight, Zhongshong City, China) that generates a rapidly evaporating mist at a rate of about $3.33 \mathrm{ml} \mathrm{min}-1$, yielding about $0.31 \mathrm{ml} \mathrm{min}^{-1}$ (9.4\%) and $0.78 \mathrm{ml} \mathrm{min}^{-1}$ (23.5\%) of ethanol. The average air speed was measured with an anemometer placed at the outlet of the tunnel and the wind speed and air volume at the inlet was reconstructed from these measurements.

Course stabilization, collision avoidance and chemical search models

Insects compound eyes lack a high resolution and acuity, but nevertheless they appear sufficient for robust flight control. The insect visual system is a specialized machinery that analyzes visual stimuli in order to produce, so called, optomotor reflexes. Many of the visual neurons found in the insect visual system are specialized in extracting motion information such as the, so called, Elementary Motion Detectors (EMDs). This is a widely studied system (Reichardt, 1961; Egelhaaf and Borst, 1993) that is able to extract directional local motion information from the visual field. The commonly used model for these neurons is based on the, so called, Reichardt correlation model (Reichardt, 1961).

The Reichardt correlation model is applied at the photoreceptor level. It computes a temporal correlation between the responses of neighboring photoreceptors $\left(I_{a}, I_{b}\right)$ separated 
by a certain distance $D$. There are two branches, the null and preferred output, which are computed independently (Fig. 5 left branch). Given a translating object from photoreceptor $a$ to its neighbor $b$ at speed $v$, the response of the correlator (Rcorr), can be explained by:

$$
\begin{aligned}
\operatorname{Rcorr}\left(I_{a}, I_{b}\right)= & \text { Preferred_output }\left(I_{a}, I_{b}\right) \\
& -N u l l \_o u t p u t\left(I_{a}, I_{b}\right)
\end{aligned}
$$

Preferred_output $\left(I_{a}, I_{b}\right)=I_{a}(t-\delta) \cdot I_{b}(t)$,

$\operatorname{Null\_ output}\left(l_{a}, l_{b}\right)=l_{b}(t-\delta) \cdot l_{a}(t)$,

Given the speed $v$ and a pixel separation of $D$,

$I_{b}(t)=I_{a}(t-D / v)$

then,

$\frac{\partial}{\partial \delta}$ Preferred_output $=0, \quad$ for $\delta=D / v$

and

$$
\frac{\partial}{\partial \delta} N u l l \_o u t p u t=0, \quad \text { for } \delta=-D / v
$$

We observe that the Reichardt correlator is optimized for a certain speed and the closer $D / v$ is to $\delta$, the higher the preferred output is (Eq. (6)); and therefore, the closer $D / v$ is to $-\delta$, the higher the null output is (Eq. (7)). The subtraction of the null branch from the preferred one results in a local motion response that is directional selective. Our system comprises four populations of EMDs tuned to drift in the four cardinal directions corresponding to rotations in the horizontal plane and changes in elevation. Course stabilization is achieved by triggering motor actions that minimize the EMD responses.

The model for collision avoidance is based on mechanisms known from the locust visual system in particular the, so called, Lobula Giant Movement Detector (LGMD). The LGMD is a wide-field neuron that specifically responds to looming,i.e. approaching, stimuli (Gabbiani et al., 2002; Hatsopoulos et al., 1995; Rind and Simmons, 1992; Schlotterer, 1977). Its firing rate depends on the velocity of and the distance to the approaching object, reaching a maximum for a specific angular size of the looming object. In previous work we have developed a model of the LGMD that was shown to allow mobile and flying robots to avoid collisions (Bermúdez i Badia and Verschure, 2004; Blanchard and Verschure, 1999; Blanchard et al., 2001). Here we present a further extension of this model that can be applied to roving and flying robots operating in complex natural environments (Fig. 5, see Bermúdez i Badia et al., 2004; Bermúdez i Badia et al., 2005). The model presented here is based on the assumption that the LGMD receives inputs from motion sensitive cells of the Reichardt type (Reichardt, 1961) in order to differentiate between expanding and contracting stimuli (Hatsopoulos et al., 1995). Our approach to the LGMD processing relies on the integration of the responses of a set of EMDs. A topographic remapping allows us to correlate the activity of pixels that are aligned radialy through the centre of the image. This process is equivalent to the integration of the responses of the EMDs that are sensitive to radial outward motion through the centre of the image. Hence, the looming sensitivity of the LGMD model emerges from its particular connectivity with the EMD neurons. These inputs are integrated and whenever its sum exceeds a given threshold it produces action potentials that will be translated into motor actions. To illustrate the operation of this obstacle avoidance model we evaluate it using a flying blimp based robot. In this case we use two cameras and two LGMD neurons are implemented to detect looming stimuli on either side of the compound visual field. Whenever a train of spikes is produced by one of the simulated LGMD cells, either left or right, it triggers an avoidance reaction in the opposite direction, performing a turn over a variable angle proportional to the strength of the response of the model LGMD. If both LGMDs respond at the same time a reverse motor action is triggered. The responses of the collision detection models overwrite the responses triggered by the stabilization model and the search system in order to prioritize avoidance reactions.

In order to evaluate the performance of the LGMD and course stabilization model we have evaluated it in a freeflight scenario using a blimp based UAV (Fig. 6, Bermúdez i Badia et al., 2005). During a four minute flight, the robot controlled by these insect based models showed obstacle detection at a mean distance of $1.69 \mathrm{~m}$ from the wall, i.e. with a minimum of $1 \mathrm{~m}$ and a maximum of $2.7 \mathrm{~m}$, the longest distance in the test room being $\sim 6.5 \mathrm{~m}$. We have shown that this model correctly deals with the trade-off between the speed of the robot and collision detection: the higher the flight speed the faster the rate of looming of stimuli in front of the robot and the sooner the LGMD model responds. In the experiments reported here we combine this biologically based optomotor system for collision avoidance with a model that supports chemical search.

The model used in our wind tunnel experiments is based on two basic behaviors observed in the moth; cross-wind casting and up-wind surge (see Introduction). Changes of the concentration of the target odor provoke transitions between these two states. In these experiments, an electronic wind vane is used for the detection of the wind direction (see 


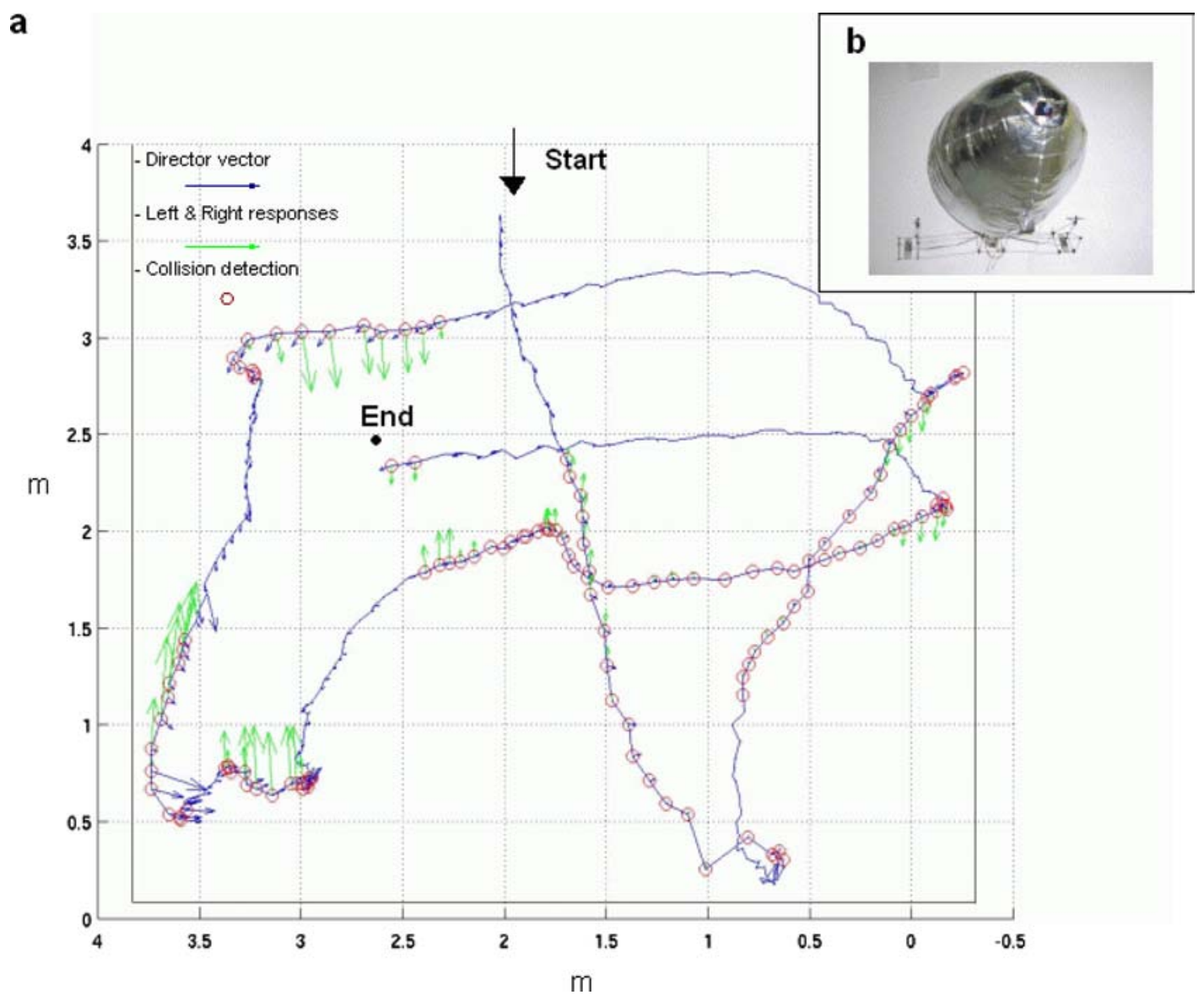

Fig. 6 (a) Trace describing one minute of free flight of the UAV with both stabilization and collision avoidance systems enabled. Blue trace: the position of the UAV every $200 \mathrm{~ms}$. Green arrows: the direction of the compensation responses to detected drift. Red dots: collision detection. (Adapted from Bermúdez i Badia et al., 2005) (b) Indoor UAV blimp platform. A mylar blimp filled with helium is propelled by 4 geared DC motors (motor: 08GS61-107.7 with gearbox 4:1 R10.0.4 both from

Methods) that allows the robot to drive upwind in case of a surge mode or to move across the wind direction in case of casting. The neural model for casting is an oscillator that triggers turns from right to left and vice versa resulting in a zig-zag trajectory (Balkovsky and Shraiman, 2002). The counter turn oscillator is running in real time at $0.043 \mathrm{~Hz}$ and is independent from the state of the robot, i.e. it is not reset when stimuli are detected. Thus the phase of the counter turn behavior is quasi random. In the cast mode, the measured wind direction is used for aligning the robot crosswind, so the search area is maximal. A contact with the plume inhibits the casting mode and switches the robot to surge mode, i.e. aligning the robot against the wind and surging as long as the stimulus is detected. The absence of an odor brings it back to casting mode (Fig. 7). In the wind tunnel experiments the course stabilization model is disabled since the mobile robot does not suffer from significant drift in its trajectory.
API-Portescap, La Chaux-de-Fonds, Switzerland, www.portescap.com; propellers are $125 \times 110 \mathrm{~mm}$ model no. 302 Paul Günther KG, Eggenfelden, Germany) fixed to a rigid carbon fiber frame $(100 \mathrm{~cm}$ long). The hull is a welded mylar blimp bag (Mobile Airships \& Blimps, Toronto Canada), that is $1.6 \mathrm{~m}$ long with a volume of $336 \mathrm{dm}^{3}$, envelope payload $237 \mathrm{~g}$. The UAV shown is also equipped with two chemosensory boards while its two cameras are visible at the front of the hull

Our model for optomotor anemotactic chemical search consists of 7082 neurons, aggregated in 97 groups, and 180 connections with 11887 synapses. It is important to note that this is a real time model of a biological neural system including a partial visual system, central motor system and olfactory system and not an abstract artificial neural network. We try to restrict ourselves to plausible implementations of biological nervous systems using standard neural models (integrate and fire, linear threshold, sigmoidal cells), while all behaviors emerges from the excitatory and inhibitory interactions among those neurons. Neither learning nor training algorithms are used and the synaptic weights are fixed.

In order to develop, manage and run simulations of this magnitude (Fig. 8), the neuronal systems simulation environment IQR was used (Bernardet et al., 2002). IQR provides the means to run large scale simulations at the speed required for the control of real-world devices. The design of the model, the control of the simulation and the acquisition of 


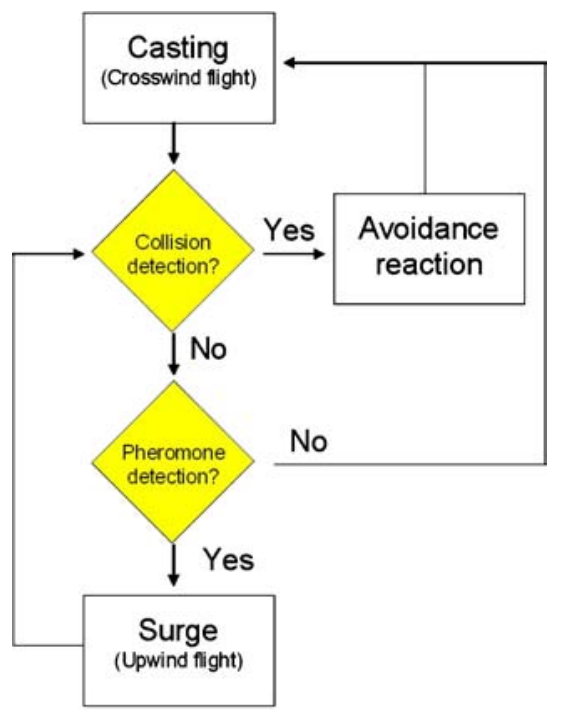

Fig. 7 Block diagram of the optomotor anemotactic chemical search algorithm applied during the experiments. The detection of a chemical stimulus, "pheromone", triggers a behavioral change from casting to surge mode, causing an upwind displacement. The collision avoidance neural model runs in parallel and overwrites the motor commands in case of imminent collision, provoking an avoidance maneuver to prevent the robot from colliding

data are done via a graphical user interface while device specific modules are written in $\mathrm{C}++$ and dynamically linked to the IQR compute engine.

\section{Results}

Before the robot experiments were performed we characterized the basic properties of our chemosensor under stan- dardized conditions, i.e. the same wind tunnel setup and the same wind and delivery conditions as used in the robot experiments. A solution of ethanol and distilled water was converted to a rapidly evaporating mist (see Methods). In addition, in order to extract the time constants of the sensor we have modulated the source using a square wave at 0.05 $\mathrm{Hz}$ (duty cycle $=50 \%$ ) note that in this case the average delivery was half of the quantity released during the continuous delivery mode described in Methods. The source was placed in the middle at the entrance of the wind tunnel (see Methods). Using a curve fitting procedure we observed a rise time of $2.0 \pm 0.77 \mathrm{~s}$ (mean $\pm \mathrm{std}, n=5$ ) and a decay time of $3.1 \pm 0.84 \mathrm{~s}$ (mean $\pm \mathrm{std}, n=9$ ) in the vector norm of the FCGs of the six sensors. Although the first order inertial curves were observed, the extracted time constants probably still contain the influence of the delivery system and airflow thus the real time constants of the sensor are probably shorter. The relatively high std may be result of these additional time constants.

In a first experiment, we mapped the distribution of ethanol in the wind tunnel while the airflow velocity was $0.67 \mathrm{~m} \mathrm{~s}^{-1}$ measured at the outlet of the wind tunnel. The source was placed in the middle at the entrance of the wind tunnel (same location as during characterization). Delivery was active and uniform (about $0.31 \mathrm{ml} \mathrm{min}^{-1}$ of ethanol, see Methods). We used subsequent measurements of 2 min per location using a single sensor. The measured response map shows that high ethanol concentrations are detected in an approximately parabolic region downwind from the source (Fig. 9(a)). These results are consistent with theoretical studies (Balkovsky and Shraiman, 2002; Shraiman and

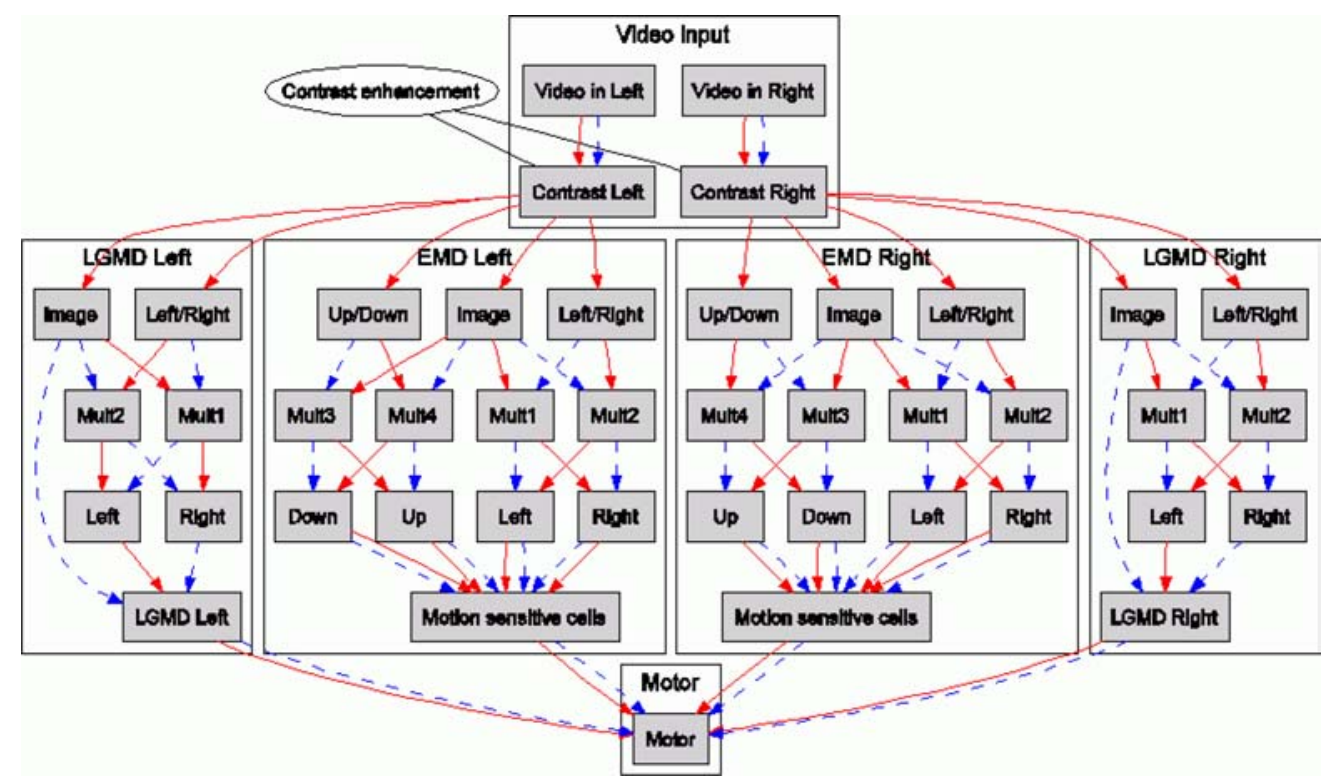

Fig. 8 Block diagram of the collision avoidance and course stabilization model used for controlling the UAV. Each block indicates one population of simulated neurons. Red arrows represent excitatory con- nections while blue lines indicate inhibition. LGMD: Lobula Giant Movement Detector, EMD: Elementary Motion Detector. The simulation runs at about $30 \mathrm{~Hz}$ using a Pentium 4 at $2.4 \mathrm{GHz}$ 


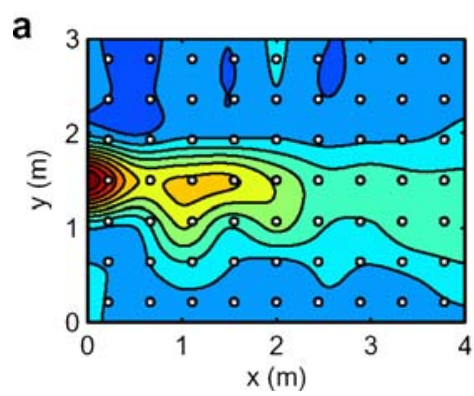

Fig. 9 Wind tunnel calibration using static measurements of ethanol. (a) Response to a $9.4 \%$ solution of ethanol in distilled water. (b) Response to distilled water only (control). The response was sequentially measured using a single sensor placed for 2 min at 9 times 7 equally spaced locations (white dots). The obtained map of time-averaged sensor responses (window size $2 \mathrm{~min}$ ) was smoothened (bi-cubic filter) and

Siggia, 2000). No detectable responses were measured when we repeated the mapping experiment with only distilled water as odor source (Fig. 9(b)) Hence, the chemosensor only responded to the dispersed ethanol. Thus, the overall setup of the wind tunnel and the response properties of the chemosensor render a reasonable response map of the ethanol concentration.

The challenge to understanding moth chemotaxis behavior is to quantify to what extent temporal features of the sensory signal change the behavior of the animal during flight. In order to better understand the nature of the instantaneous chemical signals available to the animal we visualized the chemical plume, as well as measure the instantaneous chemical signal detected by the metal-oxide chemosensor arrays (see Methods) under physiologically relevant conditions. In this way it is possible to correlate the sensor response with the qualitative properties of the chemical plume. In contrast to the conventional approach taken to machine olfaction, whereby the stimulus is deliberately controlled under known conditions, we embrace the neuroethological perspective: to understand the structure of chemical signals in natural environments and assess the signal properties in this context. We investigated chemical plumes at different flow velocities up to the maximum supporting moth chemotaxis (close to $1 \mathrm{~m} \mathrm{~s}^{-1}$ ). Low flow velocities are seen to produce a high degree of patchiness in the chemical flow field (Fig. 10(a), right), characterized by patches of relatively high concentration, interspersed by little or no chemical signal. Over larger distances these patches become less distinct, even at constant flow velocity, due to turbulent mixing of eddies governed by the Kolmogorov scale (Kolmogorov, 1941) and molecular diffusion governed by the Batchelor scale (Batchelor, 1959). Hence the chemical signal becomes less intermittent and more homogeneous as we move away from the source.

For higher flow or injection velocity (Fig. 10(b) and (c) right), greater turbulence leads to more mixing of the flow

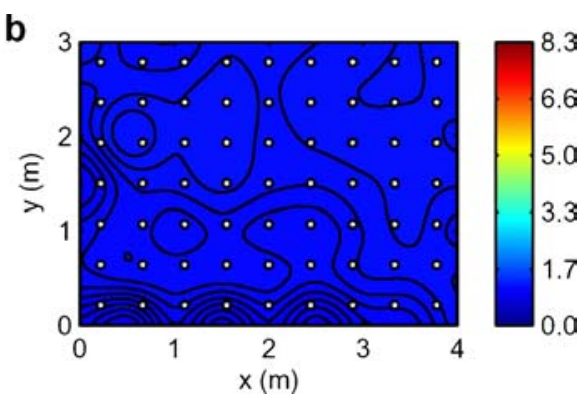

divided into 11 contours of about equal response strength. The source was placed at $(x, y)=(0 \mathrm{~m}, 1.5 \mathrm{~m})$. The wind direction is the positive $x$-axis direction. The color bar indicating response strength, as vector norm of the FCGs of the six sensors (see Methods), is representative for both maps

thus spreading the signal in space. In this case, the sensor signal contains rich temporal dynamics that correspond to the filaments of the passing chemical plume (Fig. 10(b) and (c) left). For still greater velocity, increasing levels of turbulence mix the flow locally, resulting in a less defined structure, which ultimately removes the filaments completely (Fig. 10(d) right). This results in less temporal structure in the chemosensor response since it is more uniform over time (Fig. 10(d) left). Hence, there appears to be an optimal match between the sensor dynamics and the flow velocity that allows extraction of the natural microstructure of chemical plumes.

A necessary condition to successfully locate an odor source is that the chemosensor has to be fast enough to detect differences in concentration while the robot is moving through the plume. We therefore investigated the dynamic responses of the chemosensor to ethanol by driving the robot through the wind tunnel on a zig-zag trajectory at an approximately constant translation speed of $10 \mathrm{~cm} \mathrm{~s}^{-1}$, while continuously acquiring the chemosensor response (Fig. 11). We observe that the spatial response map of the chemosensor is similar to the distribution of ethanol in the wind tunnel using static measurements (Fig. 9). The detected concentration is highest close and downwind from the source and higher concentrations are detected in a spatially constrained conical region around the (horizontal) centerline of the wind tunnel. The spatial response map obtained from averaging over 3 independent runs (Fig. 11(a)) is comparable to that from a single run (Fig. 11(b)), indicating that the observed spatial response map is repeatable across trials. Hence, these results show that the chemosensor provides a rapid and reliable measurement of the ethanol concentration while the robot is moving.

We confirmed earlier that also in our wind tunnels wind velocity crucially affects the dynamics of the odor plumes (see above). For our experiments reported here we used two wind tunnels: the Leicester wind tunnel to visualize 
Fig. 10 6-element

chemosensor array response (left column) $1.5 \mathrm{~m}$ downstream of a $30 \mathrm{ml}$ ethanol source inside a wind tunnel at different flow velocities (see methods): (a): $0.28 \mathrm{~m} \mathrm{~s}^{-1}$; (b): $0.42 \mathrm{~m} \mathrm{~s}^{-1}$; (c): $0.56 \mathrm{~m} \mathrm{~s}^{-1}$; (d): $0.70 \mathrm{~m} \mathrm{~s}^{-1}$. At each flow velocity the corresponding chemical plume, is visualized at the same height $(z=0.33 \mathrm{~m})$ as the chemosensor (right column)
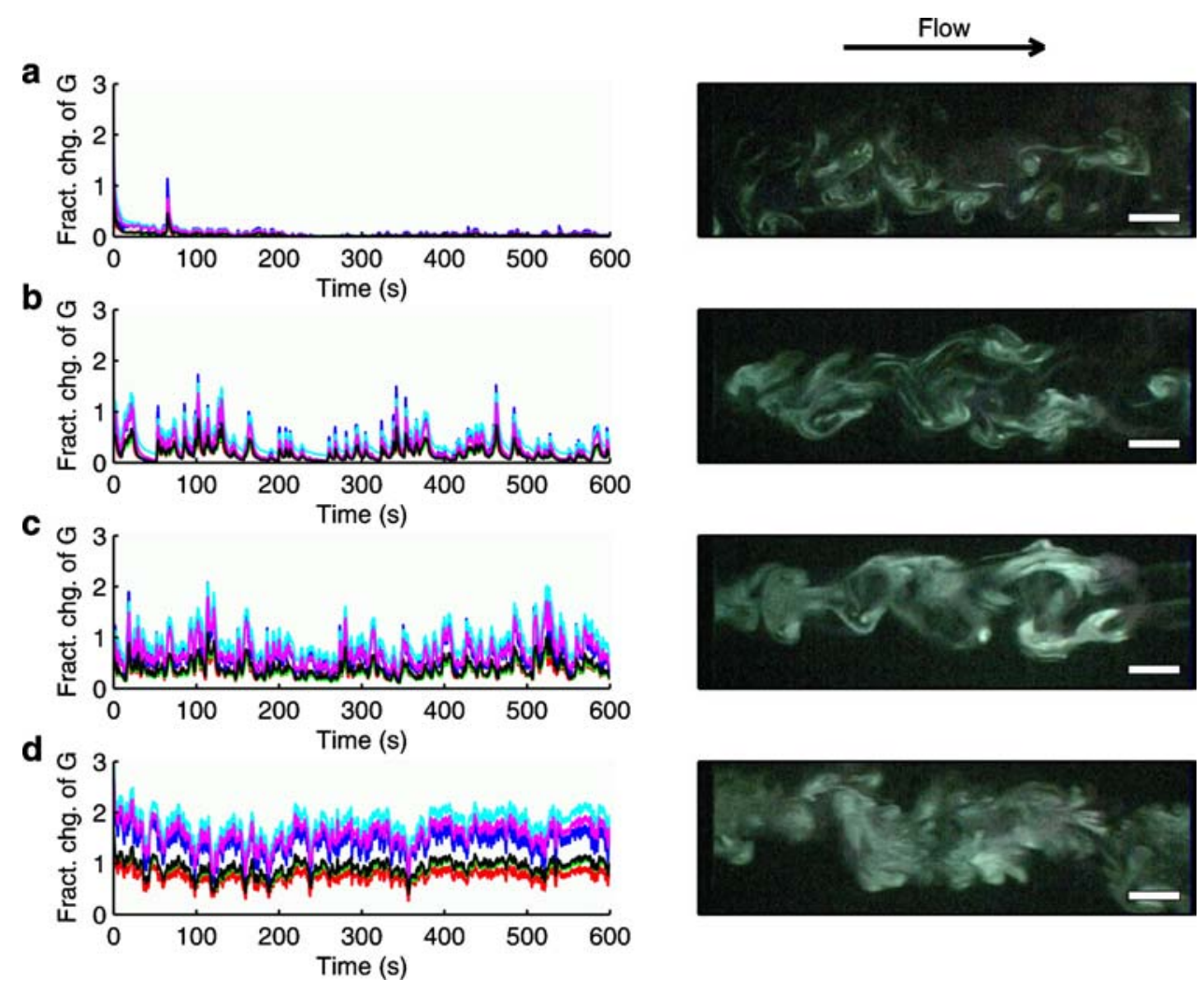

\section{b}

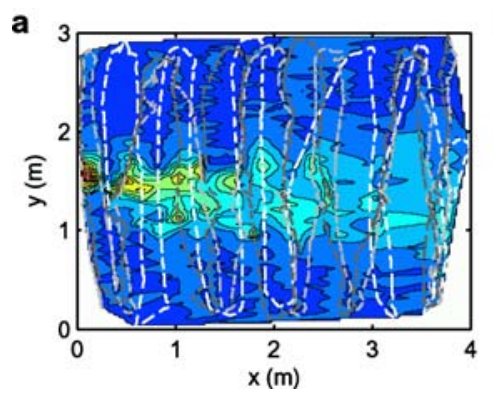

Fig. 11 Dynamic response map. Spatial distribution of the responses of the chemosensor while moving through the wind tunnel (a) averaged over three runs and (b) computed from a single run. The dashed lines indicate the track of the robot. The odor source was located at $(x, y)=(0 \mathrm{~m}$, $1.5 \mathrm{~m})$. For illustration purposes, the chemosensor response between

the plume dynamics, and the Zurich wind tunnel for the robot experiments. In order to qualitatively characterize the dynamics of the Zurich wind tunnel we compare the chemosensor response measured in the Zurich wind tunnel to that of the Leicester wind tunnel. At approximately the same wind velocity of $0.7 \mathrm{~m} \mathrm{~s}^{-1}$, a comparison of the normalized chemosensor response reveals similar dynamics in both wind tunnels, even if the robot is moving (Fig. 12(a)). This is also confirmed by comparing the power spectra of the chemosensor response (Fig. 12(b)). Hence, this indicates that the plume dynamics of the two wind tunnels are comparable and that the search experiments are performed under the complex plume dynamics depicted in (Fig. 10(d)).

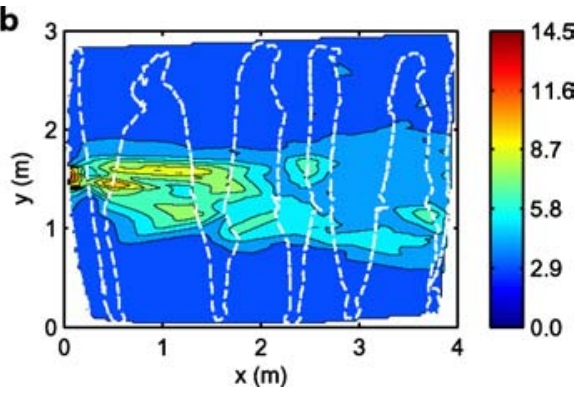

the traces was interpolated (triangle-based cubic interpolation). The resulting spatial map of the chemosensor response was divided into 11 contours. The wind direction is the positive $x$-axis direction. The airflow in the wind tunnel was $1.097 \mathrm{~m}^{3} \mathrm{~s}^{-1}$ with an average air speed of $0.667 \mathrm{~m} \mathrm{~s}^{-1}$. The robot translated at approx. $10 \mathrm{~cm} \mathrm{~s}^{-1}$

Since the chemosensor response provides a readily usable signal for the detection of ethanol, we set out to investigate the ability of the robot to perform chemical search and to locate the odor source. For each trial, the robot was placed around $3.5 \mathrm{~m}$ downwind from the source on an arbitrary $\mathrm{y}$ coordinate. The chemical search model used the wind vane sensor reading to determine the wind direction. In all trials carried out, the robot was able to find the source after an median searching time of $74.17 \mathrm{~s}$ (percentile $10 \%=47.35 \mathrm{~s}$, percentile $90 \%=170.01 \mathrm{~s}, n=37$; detailed results in Table 1, Fig. 13(a-d). Search times were not significantly different for $9.4 \%$ and $23.5 \%$ source concentrations (Wilcoxon rank sum tests for equal medians, see Table 1). We observe that 

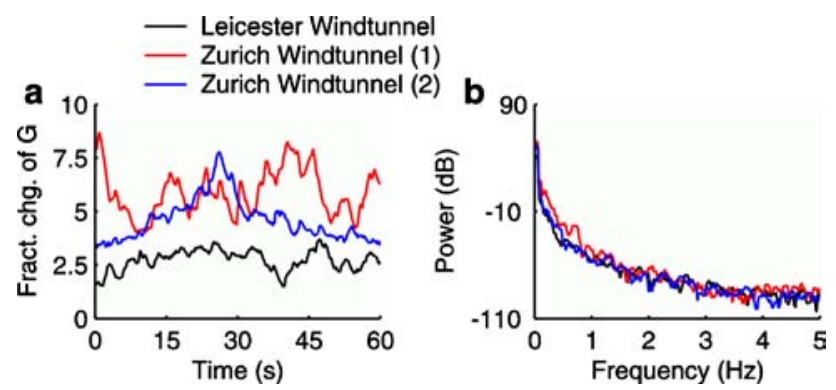

Fig. 12 Dynamics of the chemosensor response in our two wind tunnels under similar conditions. (a) Normalized response (see Methods) of the chemosensor placed $1.5 \mathrm{~m}$ downwind from the odor source in the wind tunnel at the University of Leicester (black) and in the wind tunnel at the University/ETH Zurich (red). In addition, the response measured while the robot was translating at about $10 \mathrm{~cm} \mathrm{~s}^{-1}$ is shown (blue). The wind velocity was $0.7 \mathrm{~m} \mathrm{~s}^{-1}$ and $0.67 \mathrm{~m} \mathrm{~s}^{-1}$ at the wind tunnel in Leicester and Zurich, respectively. (b) Power spectrum (estimated with the multitaper method) from the responses measured in the two wind tunnels

the system displays casting at low sensor readings and surges when the sensor reading is high. However, in the absence of wind (Fig. 13(e)) or when we replaced the ethanol mixture with distilled water (Fig. 13(f)), the robot was unable to find
Table 1 Results of the chemical search behavior of the robot excluding the optomotor system

\begin{tabular}{|c|c|c|c|c|}
\hline Ethanol source & Mode & $\begin{array}{l}\text { Percentile } \\
10 \%(\mathrm{~s})\end{array}$ & Median (s) & $\begin{array}{l}\text { Percentile } \\
90 \% \text { (s) }\end{array}$ \\
\hline \multirow[t]{3}{*}{$9.4 \%(n=17)$} & Casting & 28.37 & 39.90 & 45.72 \\
\hline & Surge & 17.91 & 33.10 & 103.66 \\
\hline & Total & 62.35 & 74.97 & 121.89 \\
\hline \multirow[t]{3}{*}{$23.5 \%(n=20)$} & Casting & 16.42 & 32.32 & 61.73 \\
\hline & Surge & 14.23 & 31.88 & 136.53 \\
\hline & Total & 45.50 & 67.83 & 174.37 \\
\hline
\end{tabular}

Results were analyzed with Lilliefors test for goodness of fit to a normal distribution. All hypothesizes about normal distribution were rejected. Wilcoxon rank sum tests for equal medians were performed to see whether there is a significant difference between search times for total search time, time of casting, and surge for an ethanol source with a concentration of $9.4 \%$ or $23.5 \%$. The test have shown show that there was no significant difference in behaviour related to concentration.

the source, showing that both airflow and chemical stimuli must be present to complete the localization behavior. We wanted to understand further the influence of concentration on behavior-our analyses show no significant difference between casting and surge time distributions for concentrations
Fig. 13 Chemical search behavior of the robot excluding the optomotor system. $(\mathrm{a}, \mathrm{b})$ Two traces of the robot with the chemical search behavior switched on (using 9.4\% ethanol, see Methods). (c, d) The same as in $(a, b)$, however, with an elevated concentration of ethanol (23.5\% ethanol). (e) Control trace without wind ( $9.4 \%$ ethanol source). (f) Control trace (source is using distilled water only). The ethanol source is denoted with a red circle. A thick line indicates an upwind surge, a thin line indicates a crosswind cast. The color of the line indicates the intensity of the sensor response. The color bar indicating response strength, as vector norm of the FCGs (see

Materials), is representative for all maps. The wind direction is the positive $x$-axis direction (average air speed of $0.667 \mathrm{~m} \mathrm{~s}^{-1}$ ). The robot translated at approx. $10 \mathrm{~cm} \mathrm{~s}^{-1}$
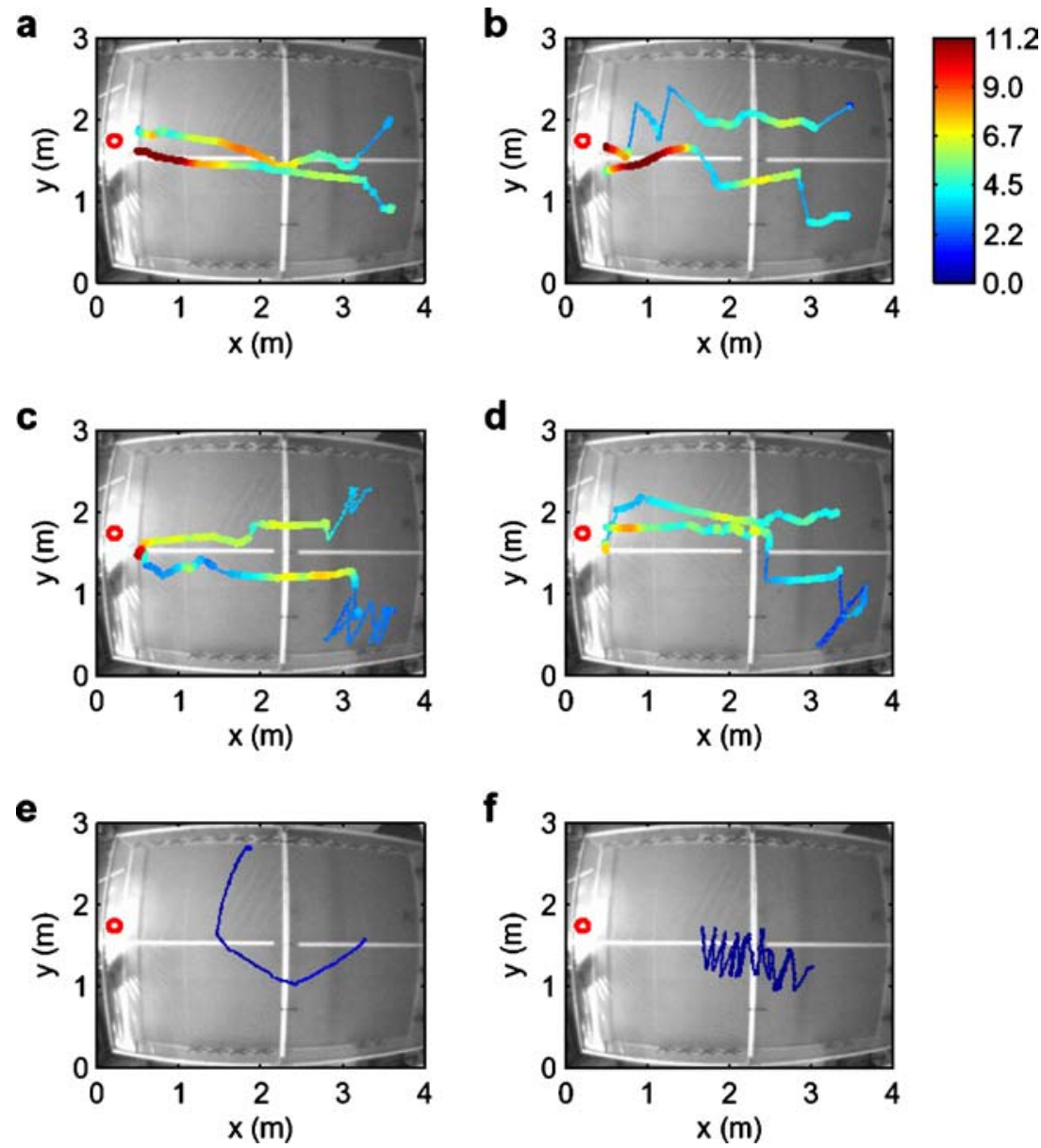
of 9.4 and $23.5 \%$. Thus we can conclude that, for the range of concentrations evaluated, the search behaviour displayed by our model is independent from the concentration.

As to be expected when only casting is triggered the robot displays a minimal overall translation against the wind direction. Hence, the moth based search behavior of the robot provides for a relatively rapid and reliable strategy to successfully localize the odor source under turbulent conditions. This demonstrates that the dynamics of the sensor, robot and control system is matched to that of the odor and plume.

Chemical search involves not only the following of an odor plume, but also the adaptive avoidance of collisions with obstacles. Hence, we combined the chemical search behavior with the collision avoidance behavior of the robot (see Methods) and introduced an obstacle placed about in the centre of the wind tunnel. Note that also the walls constitute obstacles and are marked with visual cues, i.e. black and white vertical stripes. We observed that in the case when the obstacle avoidance behavior is switched off, the robot gets stuck behind the obstacle and is unable to find the source (Fig. 14(b)). In case the LGMD based obstacle avoidance is switched on, the obstacle avoidance behavior overwrote the commands of the chemical search behavior (Fig. 14(a)). As a result avoidance reactions were displayed, after which the robot continued with the chemical search and successfully located the odor source. Thus, the combination of the obstacle avoidance behavior with the chemical search behavior renders a behavioral control structure that is robust and accurate in locating the odor source under realistic real-world conditions.

\section{Discussion}

We have presented a model of optomotor anemotactic chemical search that is based on the neuroethology of the pheromone communication system of the moth. We have shown that thin film metal oxide chemosensors show robust responses under turbulent plume conditions and can be used for robot chemical search tasks. The sensor appears to show variations in the amplitude and variance of its responses to ethanol that seem to be correlated with the plume dynamics (Figs. 9 and 10). Moreover, we have shown that the frequency content of the dynamically sampled odor plume is comparable to those of the statically measured and time integrated plume (Fig. 12). This suggests that frequency information could provide information on the odor that is invariant to movements of the sensor. When combined with the mobile platform we observe that a surge and cast model, closely resembling the behavioral analysis of moth chemical search, is effective in solving the search task. Our results showed that this model can exploit the full dynamic range of the odor plume showing its first surges, triggered by the detection of the odor at almost four meters from the odor source, i.e. practically the maximal range available in our wind tunnel (Fig. 13). On the basis of a theoretical analysis of a conceptual odor plume, it has been argued that an optimal strategy would consist of casting behavior that includes a translation at its extreme positions (Balkovsky and Shraiman, 2002). Our results suggest that this approach might not be optimal in the context of real world behavior since it would lead to an exploration strategy that is solely up wind. This would lead to the sub-optimal result that the male moth would persist in searching in a direction where the probability for successful mating is low. This illustrates the importance of analyzing individual behavioral patterns in the context of the overall behaving system. We looked at the multi-modal aspects of chemical search by combining our anemotactic chemical search system with an optomotor LGMD based collision avoidance system. The integration of these two models was based on minimal assumptions. We observed that this combined model was able to find the odor source in the presence of obstacles (Fig. 14). However, the resulting trajectory appeared suboptimal and inconsistent with the behavioral literature. The avoidance directions that were followed were independently generated from the information available to the search system not dissimilar to popular approaches in behavior based robotics (Brooks, 1986; Arkin, 1998). However, our results suggest that there could be more advanced arbitration and interaction between these behavior systems than usually assumed. In future work we will therefore analyze the interaction between the optomotor and anemotactic chemical search system in the moth in more detail.

We can compare our approach and results to those presented by a number of other groups (e.g., Kuwana et al., 1999; Lilienthal et al., 2003; Rutkowski et al., 2004). Kuwana et al. use the ElectroAntennoGram (EAG) signal of the antennas of the male silkworm moth to detect a chemical cue where the amplitude of the EAG is assumed to be proportional to the concentration. This biological sensor is applied to a small-wheeled robot (approx. $4 \times 4 \mathrm{~cm}$ (width $\times$ length)) and a simple Braitenberg vehicle type reflex based program uses two chemosensors (antennae) to control two motors in a $10 \times 4 \mathrm{~cm}$ work space. Here the chemosensors are biological while the further signal processing is strictly algorithmic. The authors show results of single runs of their system in a wind tunnel, although the robot does not use any type of wind sensor. This project provides a proof of concept for the application of biosensors, i.e. the EAGs of biological antennae, to the control of a robot, but does not contribute to our understanding of the multimodal aspects of anemotactic optomotor chemical search displayed by animals. Moreover, it is unclear how easily this approach would generalize to realworld applications. For instance, this approach has only been tested with pheromones and it is unclear how the EAG could 


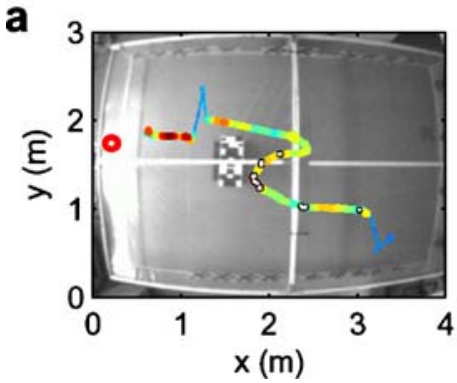

Fig. 14 Chemical search behavior of the robot in an environment containing obstacles using the LGMD-based optomotor collision avoidance system. (a) Trace of the robot with the obstacle avoidance module switched on. The robot is able find the odor source. (b) Control trace with the obstacle avoidance module switched off. An obstacle was placed in the wind tunnel (box at position $\sim(1.5 \mathrm{~m}, 1.5 \mathrm{~m}$ )). The ethanol source is denoted with a red circle. The detection of imminent

be used to process other chemical stimuli, while the robot has only been tested in a rather small area $(10 \times 4 \mathrm{~cm})$. Moreover, the biological sensor will only have a short lifetime (about 60 min under optimal conditions), while its baseline drift and non-trivial mounting and handling procedure would rather exclude its practical application.

Lilienthal et al. have presented an alternative approach (Lilienthal et al., 2003) where a large indoor mobile robot $80 \times 65 \times 55 \mathrm{~cm}$ (length $\times$ height $\times$ height) equipped with two groups of three tin-oxide chemosensors each is used for chemical search in a standard office environment $15.4 \times 5.1 \mathrm{~m}$. A sophisticated search strategy is developed to support chemical search under conditions where the airflow is below the detection limits. The proposed algorithm consists of fixed motion patterns parameterized by the expected direction of the source. Obstacle avoidance is based on odometery (clearance area around an obstacle) recalibrated by a laser range scanner. The authors show that they can trace the plume and stay in the proximity of the source. This approach lacks a clear anemotactic component since the robot is not equipped with a wind sensor. This also questions the biological relevance of the proposed algorithm. In many technical aspects our approach is similar to the one proposed by Lilienthal et al. (size of robot, search area, type of sensors). However, the time to find the source was larger by an order of magnitude, although the speed of the robot in both case was the same the search area was 6.4 times larger. However, the authors are mainly interested in the problem of plume tracing. Finding the plume itself was not the main issue and was accomplished by random search. Thus it may serve as a reference for the casting phase in our experiments.

In another approach, Rutkowski et al. study 2 and 3D chemical search using a linear Cartesian gantry to move a sensor in 2 dimensions (range $42 \times 42 \mathrm{~cm}$ ) and combine this with a treadmill (122 cm long) to add movements in a third dimension (Rutkowski et al., 2004). Although in this project

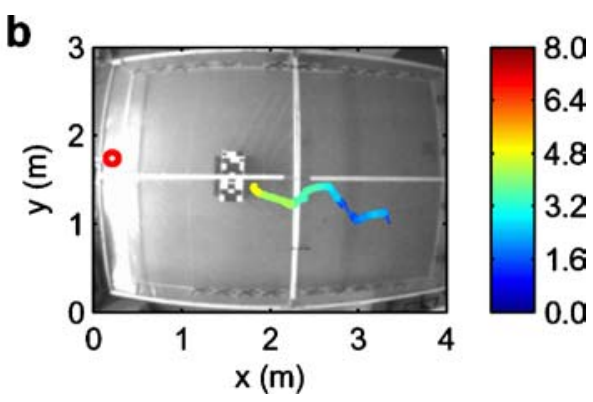

collisions is indicated with white dots. A thick line indicates an upwind surge, a thin line indicates a crosswind cast. The color of the line indicates the intensity of the sensor response. The color bar indicating response strength, as vector norm of the FCGs (see Methods), is representative for all maps. The wind direction is the positive $x$-axis direction

a 3D plume-tracking algorithm has not yet been developed, this setup provides an alternative approach to those that use mobile or flying robots. So far this setup has been used with an ionization detector. Hence, it is unclear how well it would generalize to a chemosensor. Although the flow dynamics of an ion plume is similar to that of a gas, the time constants of ionization detectors seems to be much shorter than for chemosensors. Moreover, although this setup is described as an universal platform for testing chemical search algorithms, the absence of an airflow direction sensor and the application of only one "chemosensor" under the assumption of an a priori defined fixed wind direction excludes the exploration of more realistic real-world scenarios and algorithms, including the models presented here and those proposed by Kuwana et al. and Lilienthal et al. Rutkowski et al. show an example biologically inspired algorithm demonstrating an example of successful search. However the physical nature of an ion plume and ion sensors is different than the plume generated by general stimuli, such as ethanol, used in our experiments. The static distribution of concentration measured in our experiment appears to be similar to the results of shown by Rutkowski et al. This is an important additional validation of our sensor since ion detectors are faster than chemosensors, are more stable and easier to calibrate than metal oxide sensors. Our search algorithm is based on a single translational speed and tree angles of rotation. This is consistent with the conclusion of Rutkowski et al. that only a few discrete speeds, angles of rotation and few detection levels are required to accomplish chemical search.

In addition, also other groups have used gas sensors to exploit the temporal properties of chemical plumes for robot navigation by looking at the transient sensor responses (Ishida et al., 2002) or used learning in recurrent neural networks in order to take into account the temporal sequences of sensory patterns (Duckett et al., 2001). Both of these approaches are interesting from a technical perspective but 
they do not aim at explaining the neuroethological basis of chemical search behavior.

Our results show that the current sensor and robot technology is available to construct an artificial moth that can perform complex odor source localization and mapping tasks. We are currently integrating the anemotactic chemical search system into the optomotor control systems that control our flying robots. Tests are underway that assess the ability of this system in outdoor scenarios. In addition, our results show the effectiveness of a neuroethological approach towards engineering complex real-world artifacts such as an artificial moth.

Acknowledgment This project is supported through the EU Future and Emerging Technologies programme (IST-2001-33066-AMOTH) www.amoth.org.

\section{References}

Ache, B.W. 1994. Towards a common strategy for transducing olfactory information. Semin. Cell Biol., 5(1):55-63.

Araneda, R.C., Kini, A.D., and Firestein, S. 2000. The molecular receptive range of an odorant receptor. Nat. Neurosci., 3(12):1248-1255.

Arkin, R.C. 1998. Behavior-Based Robotics. The MIT Press: Cambridge, MA.

Baker, T.C. 1990. Upwind flight and casting flight: Complimentary phasic and tonic systems used for location of a sex pheromone sources by male moths. In Proc. of the 10th International Symposium on Olfaction and Taste, Oslo, Norway, pp. 18-25.

Baker, T.C. and Kuenen, L.P.S. 1982. Pheromone source location by flying moths: A supplementary non-anemotactic mechanism. Science, 216:424-427.

Balkovsky, E. and Shraiman, B.I. 2002. Olfactory search at high Reynolds number. Proc. Natl. Acad. Sci. USA, 99(20):1258912593.

Batchelor, G.K. 1990. Small-scale variation of convected quantities like temperature in turbulent fluid. Part 1. General discussion and the case of small conductivity. J. Fluid Mech., 5:113-133.

Bau, J., Justus, K.A., and Carde, R.T. 2002. Antennal resolution of pulsed pheromone plumes in three moth species. J. Insect. Physiol., 48(4):433-442.

Bermúdez i Badia, S., Pyk, P., and Verschure, P.F.M.J. 2005. A Biologically Inspired Flight Control System for a Blimp-based UAV. In Proc. of the IEEE International Conference on Robotics and Automation (ICRA 2005), Barcelona, Spain.

Bermúdez i Badia, S. and Verschure, P.F.M.J. 2004. A Collision Avoidance Model Based on the Lobula Giant Movement Detector of the Locust. In Proc. of the International Joint Conference on Neural Networks 2004 (IJCNN'04), Budapest, Hungary, pp. 1757.

Bernardet, U., Blanchard, M.J., and Verschure, P.F.M.J. 2002. IQR: A distributed system for real-time real-world neuronal simulation. Neurocomputing, 44-46:1043-1048.

Blanchard, M.J., Rind, C., and Verschure, P.F.M.J. 2001. How accurate need sensory coding be for behavior? Experiments using a mobile robot. Neurocomputing, 38-40:1113-1119.

Blanchard, M.J. and Verschure, P.F.M.J. 1999. Using a mobile robot to study locust collision avoidance responses. International Journal of Neural Systems, 9:405-410.

Brooks, R.A.A. 1986. Robust layered control system for a mobile robot. IEEE Journal of Robotics and Automation, RA-2:14-23.
Buck, L. and Axel, R. 1991. A novel multigene family may encode odorant receptors: A molecular basis for odor recognition. Cell, 65(1):175-187.

Cardé, R.T. and Hagaman, T.E. 1979. Behavioral responses of the gypsy moth in a wind tunnel to air-borne enantiomers of disparlure. Environ. Entomol., 8:475-484.

Charlton, R.E. and Cardé, R.T. 1990. Orientation of male gypsy moths, Lymantria dispar (L.), to pheromone sources: The role of olfactory and visual cues. J. Insect Behav., 3:443-469.

Duckett, T., Axelsson, M., and Saffiotti, A. 2001. Learning to Locate an Odor Source with a Mobile Robot. In Proc. of the IEEE International Conference on Robotics and Automation (ICRA 2001), Seoul, South Korea, pp. 4017-4022.

Egelhaaf, M. and Borst, A. 1993. Motion computation and visual orientation in flies. Comp. Biochem. Physiol. Comp. Physiol., 104(4):659-673.

Firestein, S. 2001. How the olfactory system makes sense of scents. Nature, 413(6852):211-218.

Gabbiani, F., Krapp, H.G., Koch, C., and Laurent, G. 2002. Multiplicative computation in a visual neuron sensitive to looming. Nature, 420(6913):320-324.

Hatsopoulos, N., Gabbiani, F., and Laurent, G. 1995. Elementary computation of object approach by wide-field visual neuron. Science, 270(5238):1000-1003.

Haynes, K.F., Baker, T.C., Willis, M.A., and Phelan, P.L. 1985. A pulsed cloud of sex pheromone elicits upwind flight in male moths. Physiol. Entomol., 10:257-265.

Hildebrand, J.G. and Shepherd, G.M. 1997. Mechanisms of olfactory discrimination: Converging evidence for common principles across phyla. Annu. Rev. Neurosci., 20:595-631.

Ishida, H., Nakamoto, T., Moriizumi, T., Kikas, T., and Janata, J. 2001. Plume-tracking robots: A new application of chemical sensors. Biol. Bull., 200(2):222-226.

Ishida, H., Nakayama, G., Nakamoto, T., and Moriizumi, T. 2002. Controlling a gas/odor plume-tracking robot based on transient responses of gas sensors. In Proc. of the First IEEE Int. Conf. on Sensors (IEEE Sensors 2002), Orlando, USA, pp. 1665-1670.

Kennedy, J.S. and Marsh, D. 1974. Pheromone-regulated anemotaxis in flying moths. Science, 184(140):999-1001.

Kennedy, J.S., Marsh, D.M., and Ludlow, A.R. 1978. Anemotactic zigzagging flight in male moths stimulated by pheromone. Physiological Entomology, 3:221-240.

Kolmogorov, A.N. 1941. The local structure of turbulence in incompressible viscous fluid for very large Reynolds numbers. Dokl. Akad. Nauk Arm. SSR, 30:301-305.

Kuenen, L.P.S. and Baker, T.C. 1983. A non-anemotactic mechanism used in pheromone source location by flying moths. Physiological Entomology, 8:277-289.

Kuwana, Y., Nagasawa, S., Shimoyama, I., and Kanzaki, R. 1999. Synthesis of the pheromone-oriented behavior of silkworm moths by a mobile robot with moth antennae as pheromone sensors. Biosensors Bioelectronics, 14:195-202.

Lilienthal, A., Reimann, D., and Zell, A. 2003. Gas source tracing with a Mobile Robot using an adapted moth strategy. In Proc. of the AMS 2003, 18. Fachgespräch, Karlsruhe, Germany, pp. 150-160.

Ludlow, A., Perry, J., David, C., Kennedy, J., and Wall, C. 1982. A reappraisal of insect flight towards a distant source of wild-borne odor. Journal of Chemical Ecology, 8:1207-1215.

Ludlow, A.R., Kennedy, J.S., and Sanders, C.J. 1981. Guidance of flying male moths by wind-borne sex pheromone. Physiol. Entomol., 6:395-412.

Malnic, B., Hirono, J., Sato, T., and Buck, L.B. 1999. Combinatorial receptor codes for odors. Cell, 96(5):713-723.

Mombaerts, P. 1999. Seven-transmembrane proteins as odorant and chemosensory receptors. Science, 286(5440):707-711. 
Mombaerts, P. 2004. Odorant receptor gene choice in olfactory sensory neurons: The one receptor-one neuron hypothesis revisited. Curr. Opin. Neurobiol., 14(1):31-36.

Murlis, J. 1986. The structure of odor plumes. In T.L. Payne, M.C. Birch, and C.E.J. Kennedy (eds.), Mechanisms in Insect Olfaction, Clarendon Press, Oxford, UK, pp. 27-38.

Murlis, J., Elkinton, J.S., and Carde, R.T. 1992. Odor plumes and how insects use them. Annu. Rev. Entomol., 37:505532.

Murlis, J., Willis, M.A., and Carde, R.T. 2000. Spatial and temporal structures of pheromone plumes in fields and forests. Physiol. Entomol., 25:211-222.

Pearce, T.C., Chonga, K.Y., Verschure, P.F.M.J., Bermúdez i Badia, S., Carlsson, M.A., Chanie, E., and Hansson, B.S. 2004. Chemotactic search in complex environments: From insects to real-world applications. In J.W. Gardner and J. Yinon (eds.), Electronic Noses \& Sensors For The Detection Of Explosives: NATO Science Series, Kluwer Academic Pub, Dordrecht/Boston/London, pp. 181207.

Pelosi, P. 1994. Odorant-binding proteins. Crit. Rev. Biochem. Mol. Biol., 29(3):199-228.

Qian, L. and Cowen, E.A. 2002. The information content of a scalar plume-A plume tracing perspective. Environmental Fluid Mechanics, 2:9-34.

Reichhardt, W. 1961. Autocorrelation, a principle for the evaluation of sensory information by the central nervous system. In W.A. Rosenblith (ed.), Sensory Communication Contributions to the Symposium on Principles of Sensory Communication, July 19-August 1, 1959, Endicott House, M.I.T: Cambridge, M.I.T. Press, Mass., pp. 303-317.

Rind, F.C. and Simmons, P.J. 1992. Orthopteran DCMD neuron: A reevaluation of responses to moving objects. I. Selective responses to approaching objects. J. Neurophysiol, 68(5):1654-1666.

Russell, R.A. 1999. Odor Detection by Mobile Robots. World Scientific Publishing Company: Singapore.

Rutkowski, A.J., Edwards, S., Willis, M.A., Quinn, R.D., and Causey, G.C. 2004. A robotic platform for testing moth-inspired plume tracking strategies. In Proc. of the IEEE International Conference on Robotics and Automation (ICRA 2004), New Orleans, USA.

Schlotterer, G.R. 1977. Response of the locust descending movement detector neuron to rapidly approaching and withdrawing visual stimuli. Can. J. Zool., 55:1372-1376.

Shraiman, B.I. and Siggia, E.D. 2000. Scalar turbulence. Nature, 405(6787):639-646.

Turin, L. and Yoshii, F. 2002. Structure-odor relations: A modern perspective. In R. Doty (ed.), Handbook of Olfaction and Gustation, Marcel Dekker, New York, USA, pp. 275294.

Vickers, N.J. and Baker, T.C. 1994. Reiterative responses to single strands of odor promote sustained upwind flight and odor source location by moths. Proc. Natl. Acad. Sci. USA, 91(13):5756-5760.

Vickers, N.J., Christensen, T.A., Baker, T.C., and Hildebrand, J.G. 2001. Odor-plume dynamics influence the brain's olfactory code. Nature, 410(6827):466-470.

Webster, D.R. and Weissburg, M.J. 2001. Chemosensory guidance cues in a turbulent chemical odor plume. Limnol. Oceanogr., 46:1034-1047.

Willis, M.A. and Baker, T.C. 1984. Effects of intermittent and continuous pheromone stimulation on the flight behavior of the oriental fruit moth, Grapholita molesta. Physiological Entomology, 9:341-358

Zhang, X. and Firestein, S. 2002. The olfactory receptor gene superfamily of the mouse. Nat. Neurosci., 5(2):124 133.

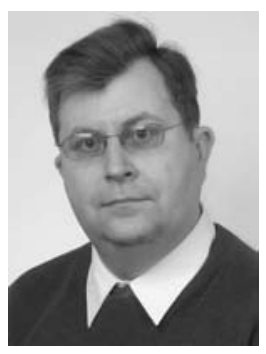

Pawel Pyk received his M.S. in Electrical Engineering (1991), M.S. in Computer Science (1994) and Ph.D. in Automatic Control and Robotics (1998), all from Silesian University of Technology, Gliwice, Poland. With his background in electronics, computer science, control engineering, system dynamics and robotics he was involved in challenging interdisciplinary research projects applied to medicine, biology, and psychology. Since 1992, he conducted his research in biomedical technology at the AO Research Institute (Davos, Switzerland). Currently he is working at the Institute of Neuroinformatics, University of Zurich/Swiss Federal Institute of Technology (ETH, Zurich, Switzerland) on biologically inspired robotics (robotics with particular focus on olfaction, chemosensory UAV and sensor integration), and on the physiological telemetry.

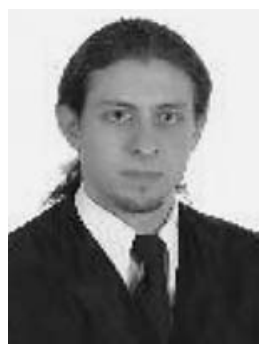

Sergi Bermúdez i Badia is a Ph.D. student at the Institute of Neuroinformatics, University of Zurich/ETH, Zurich, Switzerland since 2003. He received his B.Sc. and M.Sc. degrees in Telecommunications Engineering from the Polytechnic University of Catalonia (UPC), Barcelona, in 2003. His research interests include autonomous navigation, computational neuroscience and biologically-based modeling.

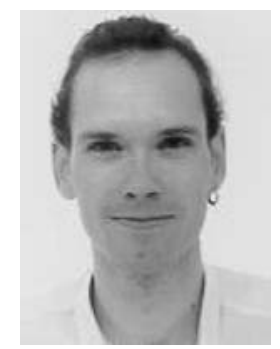

Ulysses Bernardet is a $\mathrm{PhD}$ student at the Institute of Neuroinformatics, University of Zurich/Swiss Federal Institute of Technology in Zurich (ETHZ), Switzerland. He received his M.S. in psychology, 1999, from the University of Zurich. His research interests include neural simulation, autonomous navigation and design of novel robotics platform.

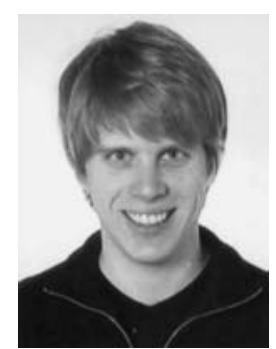

Philipp Knüsel studied physics at the Swiss Federal Institute of Technology in Zurich (ETHZ), Switzerland. Since 2002, he is a $\mathrm{PhD}$ student with Paul Verschure at the Institute of Neuroinformatics at ETHZ. His main research interests are the neuronal coding and processing of sensory information, with special emphasis on the olfactory system of insects.

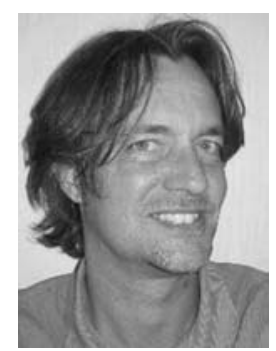

Mikael A Carlsson got basic biological training at Lund University with focus on sensory biology. He initiated his Ph.D studies in 1999 and mainly worked with neuroimaging in the moth olfactory system. After defending his thesis in 2003 at SLU he continued his work on moth olfactory processing within the AMOTH project. Since 2006 he has a post doc position at AstraZeneca in Södertälje. 


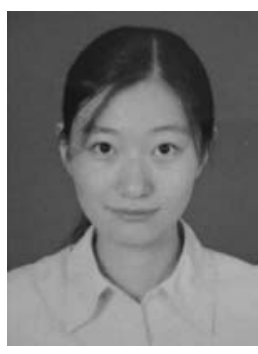

Jing Gu is a PhD Student in Engineering Department at the University of Leicester, UK. She received her Bachelors in Computer Science and Technology from Nanjing University, China. Her research interests include biologically inspired robotics, neuronal modeling, artificial intelligence, and machine learning.

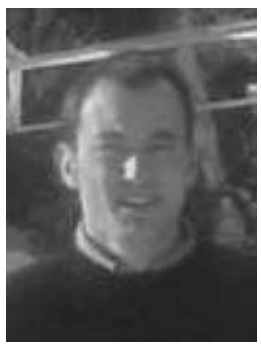

Eric Chanie is director of Research \& development of ALPHA MOS company, specialized in Smart Sensing Systems. He brings 12 years of expertise in Development \& Design of multi-sensor array technologies including sample preparation, selection and design of appropriate sensing technologies, software development, data processing techniques and artificial intelligence.

He received an engineer diploma (1993) from ENSEIRB (National Superior school of Electronic and Radiocommunication in Bordeaux, France) as well as an MBA degree (1994).

He was involved in coordination of European Research and Development projects related to artificial olfaction and is a member of the scientific council of European Network of Excellence GOSPEL (General Olfaction and sensing Projects on a European Level). He holds several patents in this field.

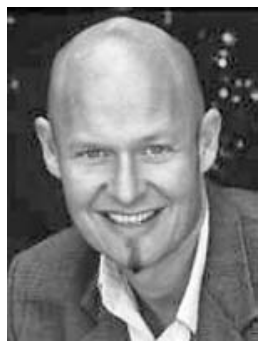

Bill S. Hansson is Director at the Max Planck Institute for Chemical Ecology in Jena, Germany. He received his BSc and $\mathrm{PhD}$ in Ecology at Lund University, Sweden. After graduating he spent a postdoc period at ARLDN, University of Arizona. In 2000 he became Professor in Lund, and in 2001 he was recruited as professor and head of division to the Swedish University of Agricultural Sciences (SLU) at Alnarp, Sweden. In 2006 he was recruited as Director of the Department of Evolutionoary Neuroethology, MPI, Jena. His research centers on the neural mechanisms underlying olfactory-driven behavior in insects, and how these systems have evolved. Systems studied include moth communication, fruit fly olfaction and giant crab olfactory evolution. He has published 125 scientific articles, several in leading scientific journals including Nature, Science, Current Biology and PNAS.

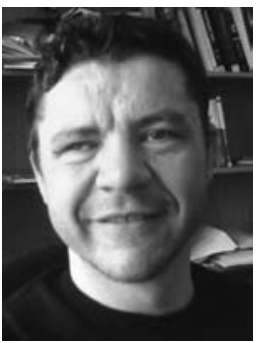

Tim C. Pearce currently holds a Lectureship in Bioengineering at Leicester University where he runs NeuroLab http://www.neurolab.le.ac.uk/ which focuses on research on computational models of olfactory information processing and their application to machine olfaction. He holds a first degree in Electronic Engineering (Honours) awarded by Warwick University and received a $\mathrm{PhD}$. from the same institution in 1997. He has since held the position of Visiting Research Assistant Professor at the Department of Neuroscience, Tufts University Medical School, Boston, USA were he worked on a DARPA supported research programme to translate principles of information processing in the biological olfactory pathway over to practical to instrumentation for chemical sensing. He currently serves as an Editorial Board member of the Journal of Neural Engineering and has been invited to teach at the Advanced European Summer School in Computational Neuroscience, Obidos, Portugal, at the 1st European School of Neuroengineering, Venice, Italy and at the Neuromorphic Engineering Workshop, Telluride, Colorado, USA on numerous occasions. He was recently elected a Fellow of the Institute of Physics and is a Junior Member of the Isaac Newton Institute for Mathematical Sciences, Cambridge.

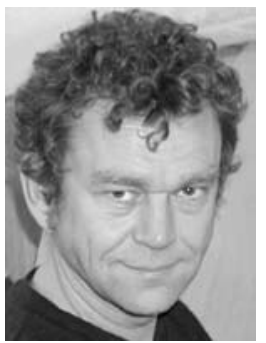

Paul F.M.J. Verschure (1962) is an ICREA research professor at the Technology Department and Foundation Barcelona Media of University Pompeu Fabra, Barcelona, Spain. He received both his Ma. and $\mathrm{PhD}$ in psychology. His scientific aim is to find a unified theory of mind, brain and body through the use of synthetic methods and to apply such a theory to the development of novel cognitive technologies. He has pursued his research at different institutes in the US (Neurosciences Institute and The Salk Institute, both in San Diego) and Europe (University of Amsterdam, University of Zurich and the Swiss Federal Institute of Technology-ETH - where he was a founding senior member of the Institute of Neuroinformatics - and University Pompeu Fabra in Barcelona). He works on biologically constrained models of perception, learning, behavior and cognition that are applied to wheeled and flying robots, interactive spaces and avatars. He has published in leading scientific journals including Nature, Science, PLoS and PNAS. Since 1998, Verschure has generated, together with his collaborators, a series of 17 public exhibits of which the most ambitious was the project "Ada: Intelligent space" for the Swiss national exhibition Expo.02, that was visited by 560000 people. 